Mon. Not. R. Astron. Soc. 000, 000-000 (0000) Printed 31 May $2021 \quad$ (MN LATEX style file v2.2)

\title{
Formation and X-ray Emission from Hot bubbles in Planetary Nebulae
}

\section{Hot bubble X-ray emission}

\author{
J.A. Toalá ${ }^{1,2 \star}$ and S.J. Arthur ${ }^{3}$ \\ ${ }^{1}$ Institute of Astronomy and Astrophysics, Academia Sinica (ASIAA), 10617 Taipei, Taiwan, R.O.C. \\ ${ }^{2}$ Instituto de Astrofísica de Andalucía, IAA-CSIC, Glorieta de la Astronomía s/n, 18008 Granada, Spain \\ ${ }^{3}$ Instituto de Radioastronomía y Astrofísica, UNAM Campus Morelia, Apartado Postal 3-72, 58090, Morelia, Michoacán, México.
}

31 May 2021

\begin{abstract}
We present a study of the X-ray emission from numerical simulations of hot bubbles in planetary nebulae (PNe). High-resolution, two-dimensional, radiation-hydrodynamical simulations of the formation and evolution of hot bubbles in PNe, with and without thermal conduction, are used to calculate the X-ray emission and study its timedependence and relationship to the changing stellar parameters. Instabilities in the wind-wind interaction zone produce clumps and filaments in the swept-up shell of nebular material. Turbulent mixing and thermal conduction at the corrugated interface can produce quantities of intermediate temperature and density gas between the hot, shocked wind bubble and the swept-up photoionized nebular material, which can emit in soft, diffuse X-rays. We use the CHIANTI software to compute synthetic spectra for the models and calculate their luminosities. We find that models both with conduction and those without can produce the X-ray temperatures and luminosities that are in the ranges reported in observations, although the models including thermal conduction are an order of magnitude more luminous than those without. Our results show that at early times the diffuse X-ray emission should be dominated by the contribution from the hot, shocked stellar wind, whereas at later times the nebular gas will dominate the spectrum. We analyse the effect of sampling on the resultant spectra and conclude that a minimum of 200 counts is required to reliably reproduce the spectral shape. Likewise, heavily smoothed surface-brightness profiles obtained from low-count detections of $\mathrm{PNe}$ do not provide a reliable description of the spatial distribution of the X-ray emitting gas.
\end{abstract}

Key words: hydrodynamics - radiative transfer — planetary nebulae:general X-rays:ISM.

\section{INTRODUCTION}

The presence of diffuse X-ray emission associated with hot bubbles in planetary nebulae $(\mathrm{PNe})$ has been confirmed by means of high-quality X-ray observations performed with the XMM-Newton and Chandra observatories (e.g., Ruiz et al. 2013, and references therein), lending strong support to the interacting stellar winds model of hot bubble formation in PNe (Kwok et al. 1978; Balick 1987). In particular, the Chandra Planetary Nebula Survey (CHAnPlaNS) is producing a statistically significant study of the X-ray emission from PNe within $1.5 \mathrm{kpc}$ of the Sun (Kastner et al. 2012; Freeman et al. 2014; Montez et al. 2015) looking for

* E-mail:toala@asiaa.sinica.edu.tw both point source X-ray emission from the central stars (CSPNe) and diffuse X-ray emission (from the hot bubbles). The results published to date have reported a diffuse X-ray emission detection rate of $\sim 30 \%$ (Kastner et al. 2012; Freeman et al. 2014). The detected objects are generally compact $\left(R_{\mathrm{HB}}<0.2 \mathrm{pc}\right.$ in radius) with closed structures, suggesting that they are young objects $\left(<5 \times 10^{3} \mathrm{yrs}\right)$, with high nebular electron densities $n_{e}>10^{3} \mathrm{~cm}^{-3}$ (as determined via their $\mathrm{H} \alpha$ line luminosities). Detailed spectral analysis of some of the detected objects indicates that the temperature of the X-ray-emitting gas lies in the narrow range $(1-3) \times 10^{6} \mathrm{~K}$ with electronic densities of the order of $1-10^{2} \mathrm{~cm}^{-3}$ (e.g., Ruiz et al. 2013; Kastner et al. 2012, and references therein). Some of the objects detected in diffuse emission can be described using the abundances of that of 
the nebular material, while others require stellar abundances (e.g., Yu et al. 2009).

The low temperatures derived from the X-ray observations contrast with expectations from adiabatic shock theory as applied to the fast stellar winds from CSPN $\left(v_{\infty}=1000\right.$ $4000 \mathrm{~km} \mathrm{~s}^{-1}$; see Guerrero \& De Marco 2013), which would indicate post-shock temperatures at least an order of magnitude higher 1 .

There have been several numerical studies that have addressed the production of diffuse X-ray emission in PNe (e.g. Mellema \& Frank 1995; Stute \& Sahai 2006; Akashi et al. 2007; Lou \& Zhai 2010), even for the case of non-isotropic wind ejections (see Akashi et al. 2008). Given that diffuse $\mathrm{X}$-ray emission is a relatively common phenomenon in planetary nebulae, any theoretical model should be able to explain all the features of the X-ray emission for a wide range of model parameters. That is, the distribution of the X-rayemitting gas, temperature, and abundances of the gas responsible for the diffuse emission. The main sequence mass of the central star sets the timescale for the mass loss during the AGB stage and thus determines the density distribution of the circumstellar medium around the CSPN. In the postAGB stage the stellar parameters are determined by the mass of the central hot object that remains after the hydrogen envelope has been expelled. Previous 1D numerical work by Akashi et al. (2007) and Steffen et al. (2008) has shown that it is important to take into account the time history of the stellar wind as it increases in velocity and decreases in mass-loss rate over the few hundred years after the AGB envelope is ejected. In order to explain the low X-ray temperatures, either the X-rays come from wind shocked while the velocity was still low $\left(v_{\mathrm{w}}<500 \mathrm{~km} \mathrm{~s}^{-1}\right)$, or some physical process operates to produce large quantities of gas at about a million degrees even when the stellar wind velocity is higher than $1000 \mathrm{~km} \mathrm{~s}^{-1}$. Soker (1994) suggested that thermal conduction could contribute to the production of the diffuse X-ray emission, and that the soft X-rays come from a conduction layer of evaporated nebular gas adjacent to the hot bubble, rather than from the shocked fast stellar wind.

The one-dimensional (1D) radiation-hydrodynamic simulations presented in Steffen et al. (2008) provided a coherent view of the formation and X-ray emission from $\mathrm{PNe}$ by considering the detailed time evolution of the central star parameters and ionizing photon flux from the asymptotic giant branch (AGB) through to the post-AGB phase. These authors included heat diffusion in their simulations and considered both classical and saturated thermal conduction (see, e.g., Spitzer 1962). They used two different formalisms for limiting the electron mean free path: one that depends essentially on the numerical resolution and can be interpreted as inefficient conduction, and the other, which defaults to the saturation heat flux wherever the classical heat flux exceeds the saturated value, and represents efficent conduction. They found that their simulations reproduce the plasma temperatures and luminosities as long

\footnotetext{
1 For an adiabatic shock in a free-flowing stellar wind with velocity $v_{\infty}$, the post-shock temperature is $k_{\mathrm{B}} T_{\mathrm{S}}=3 \mu m_{\mathrm{H}} v_{\infty}^{2} / 16$, where $\mu m_{\mathrm{H}}$ is the mean mass per particle and $k_{\mathrm{B}}$ is Boltzmann's constant (see, e.g., Dvson \& Williams 1997).
}

as conduction is included. However, they favoured the numerical results from their efficient conduction method, arguing that the low-efficiency conduction method results in limb-brightened synthetic X-ray surface brightness profiles, which did not match some observations at the time. This group has recently extended its numerical simulations to study the X-ray emission from PNe with hydrogen-deficient winds (Sandin et al. 2016).

In Toalá \& Arthur (2014, hereafter Paper I) we presented high resolution two-dimensional (2D), radiationhydrodynamical simulations of the formation and evolution of hot bubbles inside PNe for different initial stellar masses. We used the stellar evolution models at solar metallicities $(Z=0.016)$ from Vassiliadis \& Wood (1993) for the AGB phase and the corresponding models from Vassiliadis \& Wood (1994) for the stellar parameters in the post-AGB phase. We computed the stellar wind parameters for the post-AGB phase with the WM-basic code (Pauldrach et al. 1986; Pauldrach 1987; Pauldrach et al. 1994, 2001, 2012). We found that the fast wind/AGB interaction zone is unstable and that the interface between the hot bubble and the swept-up photoionized shell corrugates and forms clumps and filaments. Even without thermal conduction, hydrodynamical ablation and photoevaporation of the dense clumps leads to turbulent mixing of cooler material into the hot gas. When thermal conduction is included in the simulations, heat diffuses from the hot shocked gas in the interior of the bubble into the surrounding cooler photoionized shell. Dense material from the shell is heated and expands, and the corrugated nature of the interface increases the efficiency of this process. In both scenarios, the net result is a significant amount of material at intermediate temperatures and densities, which originated from the clumps formed at the unstable interface.

In this paper, we use the results from Paper I to generate synthetic X-ray emission, and study the time-variation of the X-ray spectra, luminosities, and plasma temperatures for models with different initial stellar masses and models with and without thermal conduction. The structure of the present paper is as follows: in Section 2 we describe the methodology followed, and the results are presented in Section 3 . In Section 4 we compare our results with observations. We present the discussion of our results in Section 5 and summarize our findings in Section 6

\section{METHODOLOGY}

We start from the 2D, axisymmetric, radiationhydrodynamic numerical simulation results of the formation and evolution of hot bubbles in PNe from Paper I and calculate their corresponding synthetic X-ray emission. We use the models corresponding to stellar initial masses $\left(\mathrm{M}_{\mathrm{ZAMS}}\right)$ of $1,1.5,2$, and $2.5 \mathrm{M}_{\odot}$ and consider the cases with and without thermal conduction. These models correspond to final white dwarf (WD) masses of 0.569, 0.597, 0.633 , and $0.677 \mathrm{M}_{\odot}$. Models with initial masses of 3.5 and $5 \mathrm{M}_{\odot}$ are not used as they create only ionized-bounded PNe, which never become optically thin (see Villaver et al. 2002; Perinotto et al. 2004, Paper I). For simplicity, and consistency with Paper I, we will refer to the models used here by a combination of their initial mass and their final 

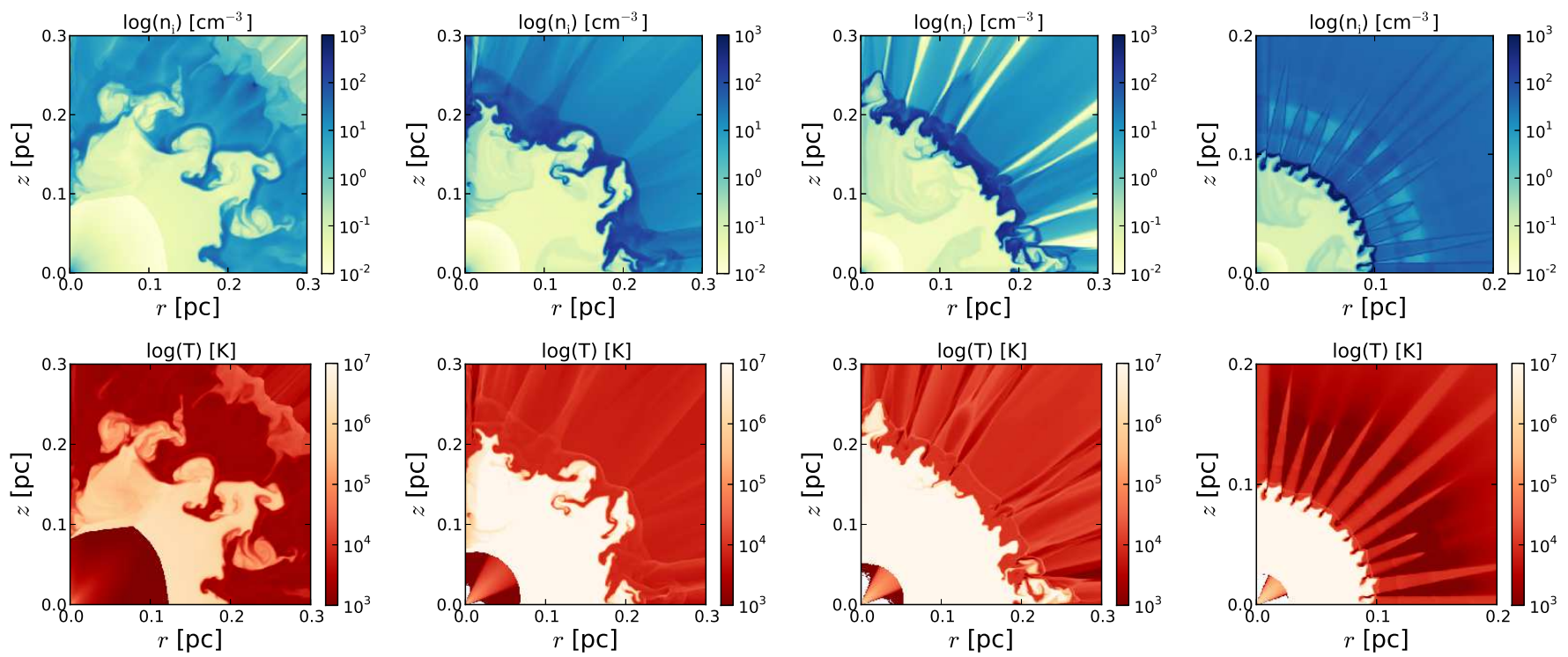

Figure 1. Total ion number density (top panels) and temperature (bottom panels) in the $r-z$ plane as presented in Paper I. Columns (from left to right) correspond to the 1.0-0.569, 1.5-0.597, 2.0-0.633, and 2.5-0.677 models without thermal conduction at 6500, 7000, 4500 , and 1500 yr after the onset of the post-AGB phase, respectively.
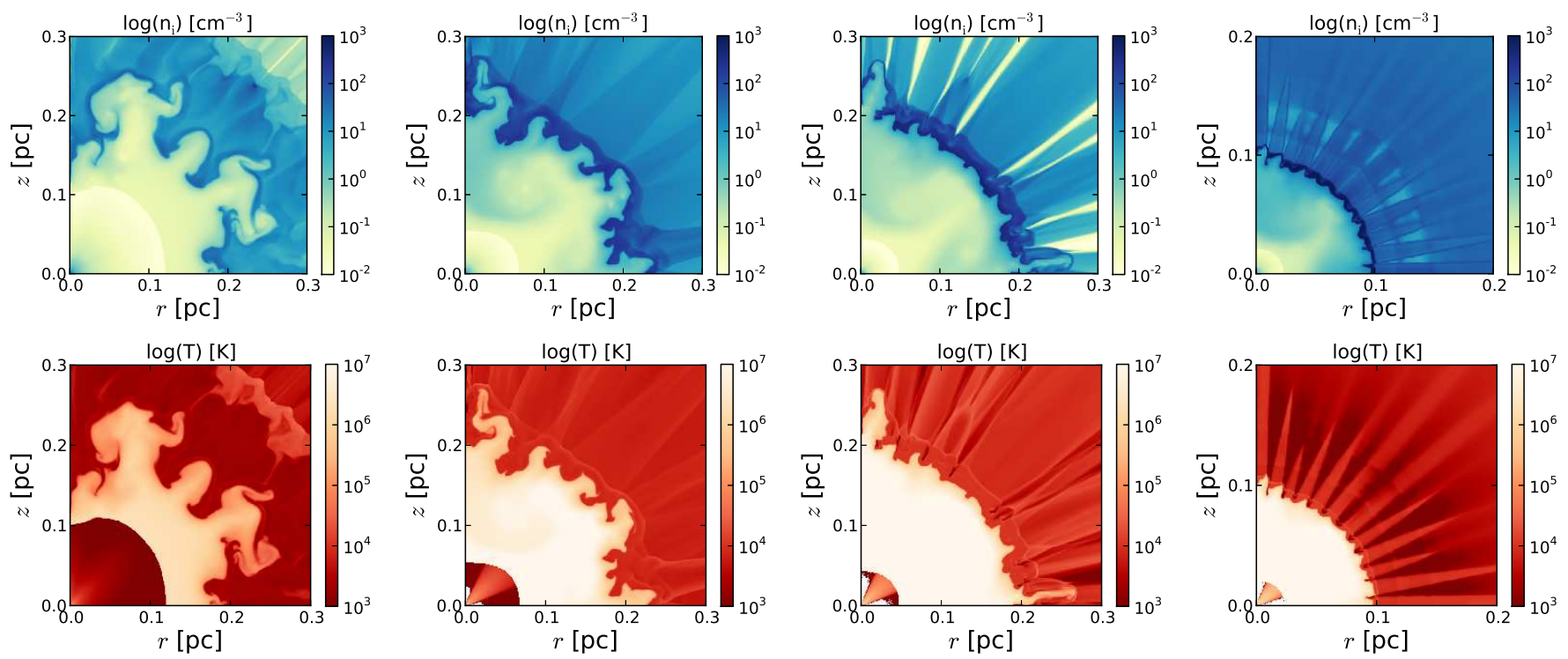

Figure 2. Same as Figure 1 but for cases with thermal conduction. Columns (from left to right) correspond to the 1.0-0.569, 1.5-0.597, 2.0-0.633, and 2.5-0.677 models at 6500, 7000, 4500, and $1500 \mathrm{yr}$ after the onset of the post-AGB phase, respectively.

WD masses. For example, the stellar model with initial mass of $1 \mathrm{M}_{\odot}$ and final WD mass of $0.569 \mathrm{M}_{\odot}$ will be labeled as 1.0-0.569. Thus, the labels of the other models are 1.5-0.597, 2.0-0.633, and 2.5-0.677.

As an illustration of the density and temperature distributions that resulted from the simulations presented in Paper 12, we show in Figures 1 and 2 the total ion num-

\footnotetext{
2 While writing the present paper, we detected an error with our conduction subroutine and have recalculated all the models from Paper I. The results are qualitatively similar and we present the new calculations here in Figures 1 and 2
}

ber density and gas temperature in the $r-z$ plane for all the models, at the point where the hot bubble in each model has an average radius of $\sim 0.2 \mathrm{pc}$, except for the $2.5-0.677$ model which corresponds to the time when the hot bubble has an averaged radius of $0.1 \mathrm{pc}$. As described in detail in Paper I (see Sections 4.1 and 5 of that paper), instabilities, which develop early on in the interaction of the fast wind with the nebular gas, play an important rôle in the subsequent evolution of the hot bubble. Dense clumps and filaments resulting from the instabilities in the swept-up shell present a large surface area. Heat diffusion from the hot, shocked gas into the cooler clumps and filaments lowers the temperature throughout the hot bubble and also results in 

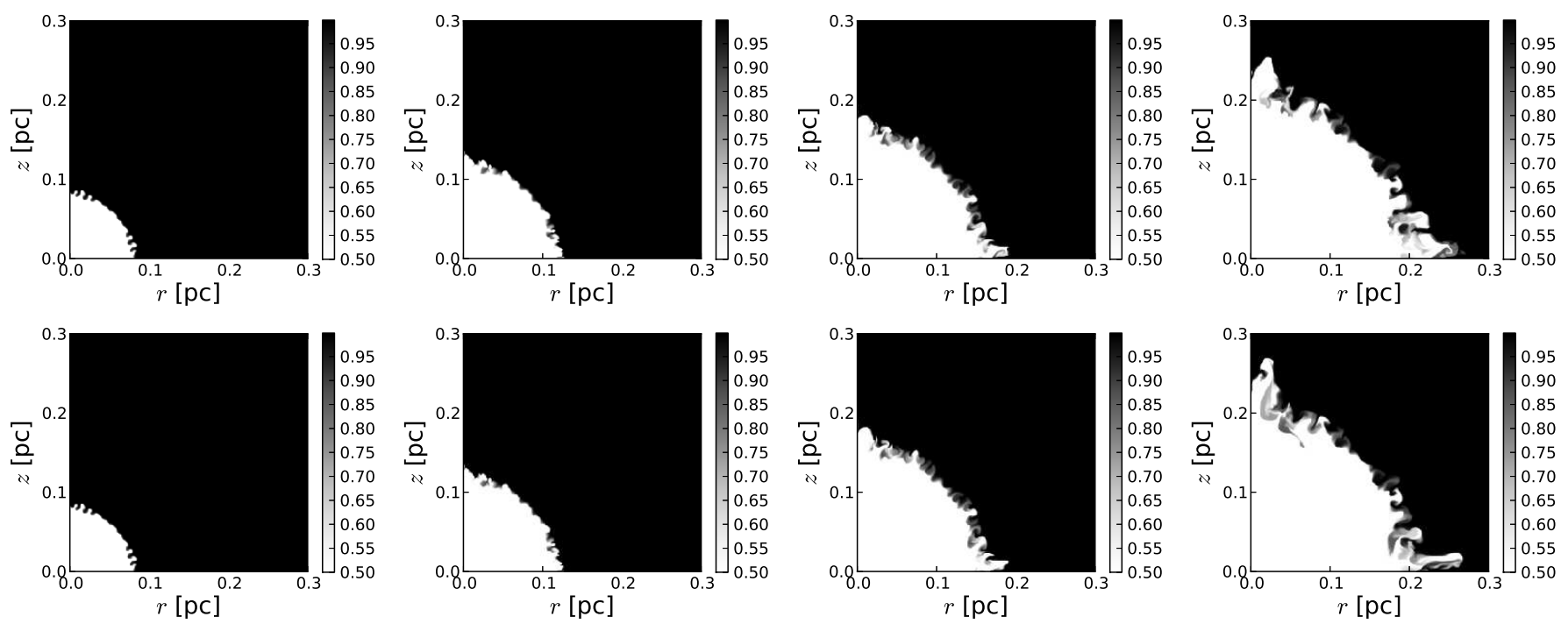

Figure 3. Advected scalar $s$ value in the $r-z$ plane for the 2.0-0.633 models. Top row: models without conduction; bottom row: models with thermal conduction. From left to right, the panels correspond to mean hot bubble radii $0.05,0.10,0.15$, and 0.20 pc at 1100,2100 , 3100 , and $4100 \mathrm{yr}$ after the onset of the post-AGB phase, respectively. Scalar values of $0.5<s<=1$ (shown in greyscale) correspond to predominantly nebular (i.e., expelled AGB envelope) material, while values of $0<=s<0.5$ (white) correspond to predominantly fast stellar wind material.

hot $\left(T_{\mathrm{e}} \sim 10^{6} \mathrm{~K}\right)$, dense $\left(n_{\mathrm{e}} \sim 1.0 \mathrm{~cm}^{-3}\right)$, evaporated nebular material expanding inwards into the hot bubble (see Fig. 2). Even in the absence of conduction, photoevaporation and hydrodynamic ablation of the clumps and filaments form turbulent mixing layers of shocked, heated nebular gas $\left(T_{\mathrm{e}} \sim\right.$ a few $\left.\times 10^{6} \mathrm{~K}\right)$ at the outer, corrugated boundary of the hot bubble (see Fig. 1).

To understand the evolution of the mixing layers, we use an advected scalar, $s$, which is assigned the value of $s=1$ in the nebular (i.e., expelled AGB envelope) material prior to the onset of the fast wind. The fast wind is injected into the grid with a scalar value of $s=0$. The scalar enables us to see how far the evaporated nebular material penetrates into the hot bubble, in the models with conduction, and the extent of the turbulent mixing layers, in the models without conduction. Intermediate values of the scalar develop as a result of mixing within the Eulerian cells of the 2D simulation, particularly in regions with strong pressure variations, such as cooling zones, and also in the region of hydrodynamic instabilities (see Paper I). Figure 3 shows the evolution of the scalar for the 2.0-0.633 models with and without thermal conduction. The final panels in each row of Figure 3 should be compared to the corresponding panels in Figures 1 and 2. when the mean hot bubble radius is $0.2 \mathrm{pc}$. Other initialfinal mass models are similar.

Figure 3 shows that at early times the distribution of the scalar in the models with and without conduction is the same, and shows how hydrodynamic instabilities break up the swept-up dense shell. As the evolution proceeds, thermal conduction evaporates material from the corrugated inner wall of the dense shell, which begins to expand inwards into the hot, shocked wind bubble (Fig. 3. lower right-hand panel). On the other hand, the hydrodynamic mixing layers around the clumps and filaments in the models without conduction are closely coupled to these structures and do not expand greatly with time. In the panels of Figure 3 computational cells containing mainly fast stellar wind material $(s \leqslant 0.5)$ are coloured white, while those containing mainly mixed nebular material $(s>0.5)$ are shown with a greyscale. Figure 3 shows that without conduction, the hot, shocked wind penetrates further into the dense, swept-up nebular shell.

The expansion of the hot bubble can be characterized by its mean radius as a function of time. This is shown in Figure 4 for each of the models. For a given mass model, the hot bubble radii for the simulations with and without conduction follow the same expansion except for the lowest mass models (1.0-0.569). In these particular models, there is more radiative cooling in the models with conduction and consequently less thermal energy available to drive the expansion.

The hot bubbles continue to expand even after the stellar wind mechanical luminosity begins to decrease. Unfortunately, we are not able to follow the expansion of the 2.00.633 and 2.5-0.677 models much beyond the turnover point in the wind luminosity. This is because the ram pressure of the fast wind becomes smaller than the thermal pressure of the hot bubble and the inner shock collapses back down towards the star. This causes the computational timestep to become extremely small and the calculation effectively comes to a halt.

A comparison with the expansion of equivalent 1D models, depicted in Figure B1 shows very similar behaviour. The lowest mass models (1.0-0.569) expand slightly faster in the $1 \mathrm{D}$ case, while the higher mass models expand slightly slower but there are no great differences.

\subsection{Differential emission measure}

The first step towards calculating the X-ray emission from our simulations is to compute the differential emission measure (DEM), defined in this work for a given temperature 

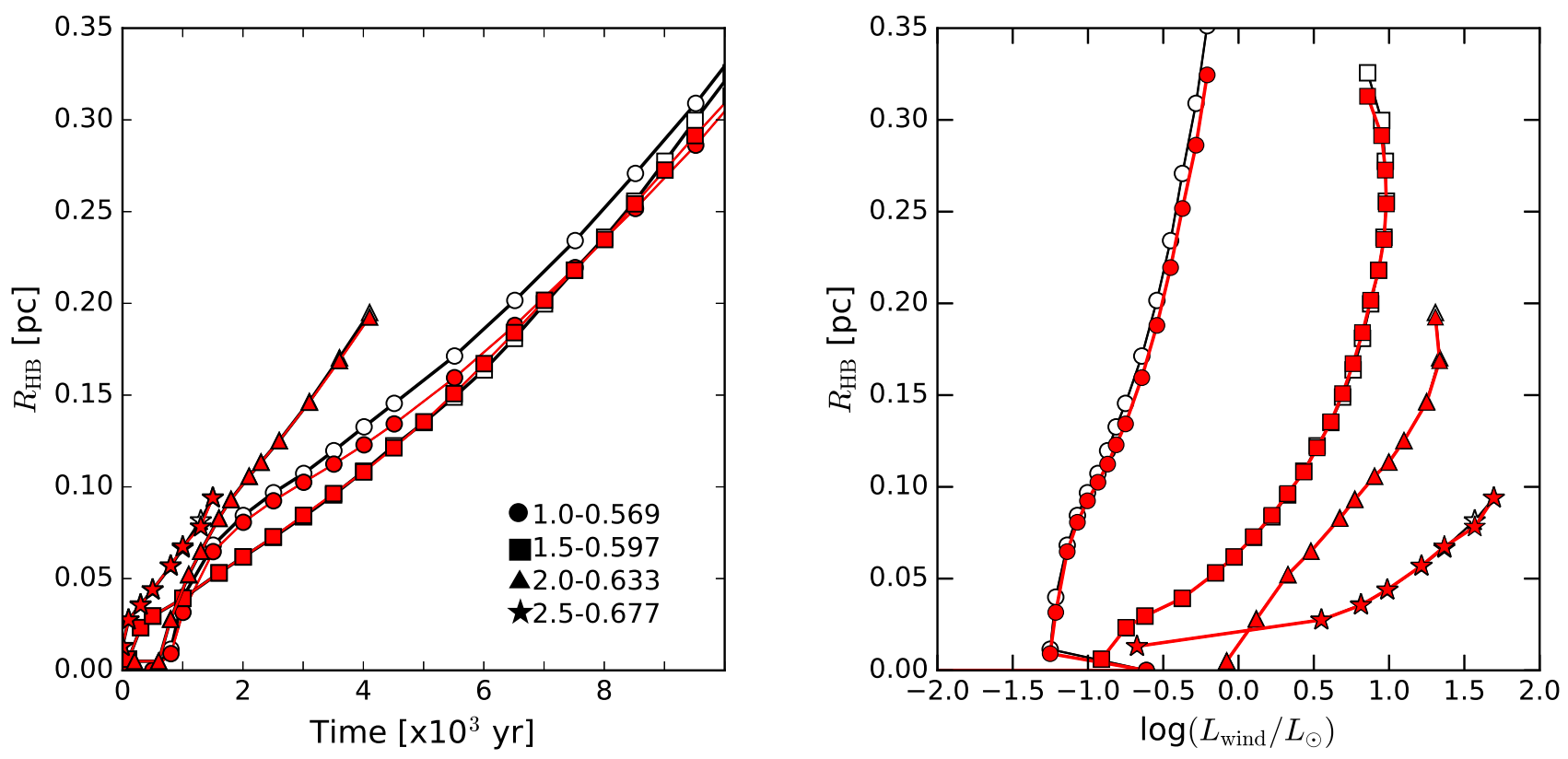

Figure 4. Mean radius of the hot bubble $\left(R_{\mathrm{HB}}\right)$ as a function of time (left panel) and as a function of stellar wind mechanical luminosity (right panel) for each stellar model, with and without thermal conduction. Filled symbols are for the models that include thermal conduction, while open symbols are for the models that do not.
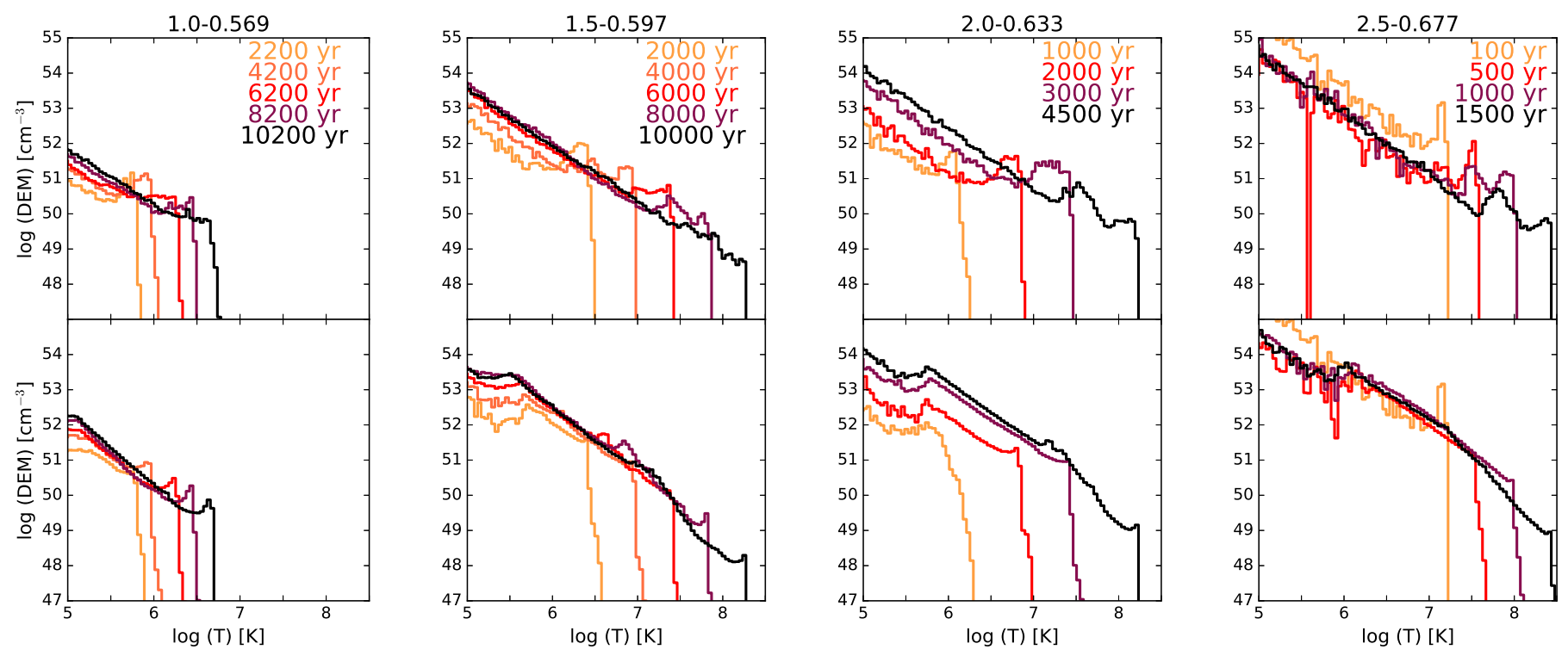

Figure 5. Evolution with time of the differential emission measure (DEM) of hot bubbles for the four stellar models considered in this work. Upper and lower panels correspond to cases without and with thermal conduction, respectively. Times are marked with different colours.

bin as

$$
\operatorname{DEM}\left(T_{\mathrm{b}}\right)=\sum_{k, T_{k} \in T_{\mathrm{b}}} n_{\mathrm{H}} n_{\mathrm{e}} \Delta V_{k}
$$

where $n_{\mathrm{H}}$ and $n_{\mathrm{e}}$ are the hydrogen vand electron number density in cell $k$, respectively, $\Delta V_{k}$ is the volume of cell $k$ and the sum is performed over cells with gas temperature falling in the bin whose central temperature is $T_{\mathrm{b}}$. In practice, we use logarithmic binning in the temperature range $\log (T)=5$ to $\log (T)=9$ in intervals of 0.04 dex (i.e., 100 bins). Lower temperatures are not taken into account in calculating the DEM, since their contribution to the X-ray emission will be negligible.

As a result of the evolution hot gas inside the PN 
(i.e., the temperatures, densities and volume), the DEM histogram of a given model changes with time. We show in Figure 5 the time evolution of the DEM for all the models used in this work. The maximum temperature reached by each DEM curve corresponds to the immediate post-shock temperature at the given time. The increase in the maximum temperature with time is a consequence of the increasing fast-wind velocity. Note that all the inner stellar wind shocks in our models are adiabatic and hence the post-shock temperature dependes only on the square of the wind velocity and are independent of the shock position. A different treatment of the conduction could lead to a radiative innershock whose post-shock temperature is position dependent (e.g., Steffen et al. 2008).

In particular, the case of the 1.0-0.569 model does not show a contribution at temperatures above $10^{7} \mathrm{~K}$ because even after $\sim 10,000 \mathrm{yr}$ the post-AGB wind velocity is still $<800 \mathrm{~km} \mathrm{~s}^{-1}$ and does not produce hotter gas (see Fig. A1 in Appendix A). For more massive models, higher stellar wind velocities are reached on shorter time scales and so their DEM histograms have significant contributions at temperatures $T \sim 10^{8} \mathrm{~K}$. It can be also seen in Figure 5 that as the stellar wind parameters evolve, the relative contribution from the higher temperatures diminishes because the mass-loss rate, and hence the post-shock density are falling.

On the other hand, the DEM distributions of the models with thermal conduction (Fig. 5 bottom panels) show a plateau at lower temperatures and the contribution at higher temperatures for the late time evolution is lower than in their counterparts without conduction. This is due to the diffusion of heat from the hot gas into the surrounding cooler dense shell. In general, the models with thermal conduction have slightly higher DEM values than those without thermal conduction, particularly for the $10^{6} \mathrm{~K}$ gas. As a result, the fluxes and X-ray luminosities of the models with thermal conduction will be higher than in cases without conduction.

For comparison, we show in Appendix B the DEM distributions obtained from 1D simulations for the 1.5-0.597 models with and without conduction. The $1 \mathrm{D}$ case without conduction shows a sparse, discontinuous DEM distribution, quite different to the $2 \mathrm{D}$ case. The $1 \mathrm{D}$ case with conduction does not have such a large component at temperatures $\log T<6.0$ as its $2 \mathrm{D}$ counterpart because the amount of gas in the conduction layer is smaller (in 2D the surface area of the conduction layer is much larger due to the clumps and filaments formed in these simulations).

\subsection{Plasma Temperatures}

An important quantity that can be readily obtained from the DEM distributions is the average temperature of the hot gas $\left(T_{\mathrm{A}}\right)$. Observations of diffuse X-ray emission generally report a characteristic temperature $\left(T_{\mathrm{X}}\right)$ for the emitting gas in $\mathrm{PNe}$, which is obtained by fitting a single temperature model to the observed spectrum. The average temperature we calculate from our models should be a reasonable estimate to the observationally derived characteristic temperature if a single-temperature model is indeed appropriate (e.g., $T_{\mathrm{A}} \approx T_{\mathrm{X}}$ ).

We define an emissivity weighted averaged temperature,

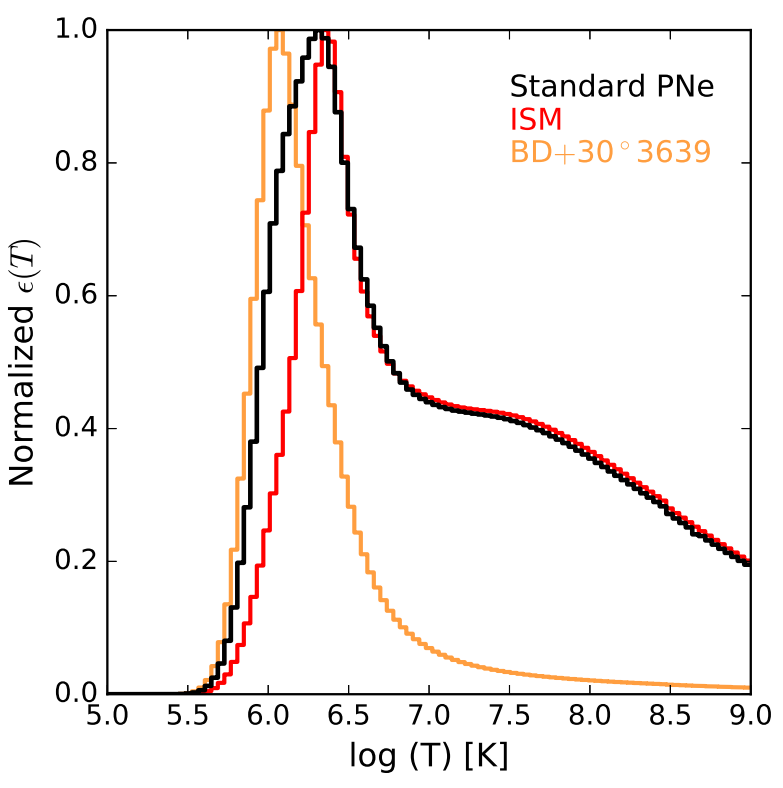

Figure 6. Normalized emission coefficient $\epsilon(T)$ (see Eq. 3) for the 0.3-2.0 keV energy range computed for the standard PN abundance set used in this work (black solid line). Other emission coefficients computed for different abundance sets are also shown.

$T_{\mathrm{A}} \equiv\langle T\rangle$, as:

$$
T_{\mathrm{A}}=\frac{\int \epsilon(T) \operatorname{DEM}(T) T d T}{\int \epsilon(T) \operatorname{DEM}(T) d T},
$$

where $T$ is taken to be the electron temperature and

$$
\epsilon(T)=\int_{E_{1}}^{E_{2}} \epsilon(T, E) d E
$$

is the emission coefficient integrated over the energy band $E_{1}=0.3 \mathrm{keV}$ to $E_{2}=2.0 \mathrm{keV}$ for the standard planetary nebula abundances used in this work (see Table 1 in Paper I). Figure 6] shows that, for standard planetary nebula abundances, the emission coefficient is sharply peaked around $\log (T)=6.3$ and drops off very steeply towards lower temperatures. Since, in general, the $\operatorname{DEM}(\mathrm{T})$ rise towards lower $\mathrm{T}$, the emissivity weighted average temperature will always be close to the peak temperature of the emission coefficient.

Figure 6] shows why it is not surprising that most detections of diffuse X-ray emission from extended sources (PNe, Wolf-Rayet bubbles, and stellar wind bubbles) report soft $\mathrm{X}$-ray temperatures of at most a few million degrees. For different abundance sets, the behaviour of the emission coefficient is similar but with different intensities: a narrow peak between $1-3 \times 10^{6} \mathrm{~K}$ that falls off sharply towards both lower and higher temperatures. Lower metallicity, such as for the ISM abundance set, moves the emission coefficient peak towards higher temperatures $(\log T=6.4)$. The peak is narrower and corresponds to $94 \%$ of that the PN abundance set, due to the fact that metals such as carbon and oxygen are less important. Higher metallicity, like that of the [WC]type central star of $\mathrm{BD}+30^{\circ} 3639$ (see Marcolino et al. 2007), leads to a narrow peak at lower temperature $(\log T=6.1)$ and a steep fall off towards higher temperatures since there is no hydrogen to produce free-free emission above $\log T=6.5$. 


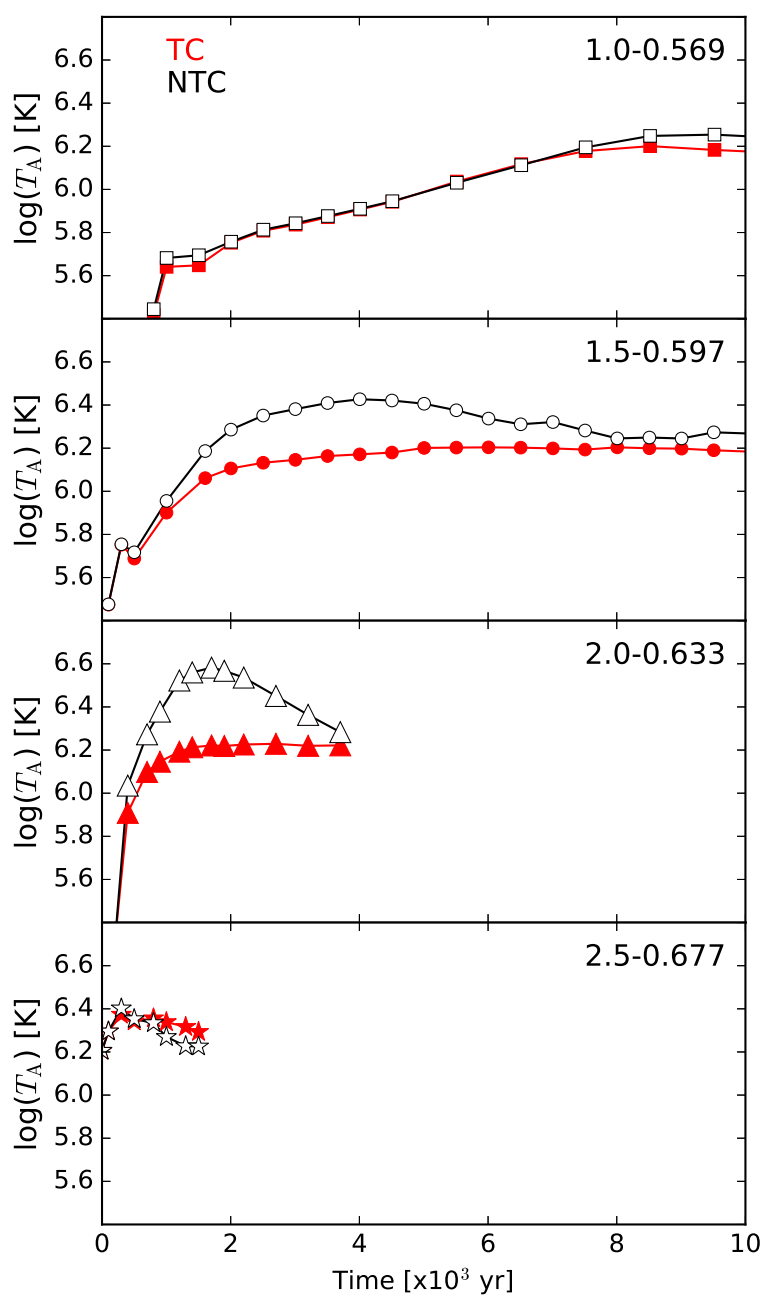

Figure 7. Average plasma temperature $T_{\mathrm{A}}$ in the hot bubbles for different models. Filled and open symbols represent models with (TC) and without thermal conduction (NTC), respectively. The final plotted times correspond to $\log \left(T_{\text {eff }}\right)=4.49,5.12,5.18$, and 5.02 for the $1.0-0.569,1.5-0.597,2.0-0.633$, and 2.5-0.677 models, respectively.

This last case, has coefficient intensities almost an order of magnitude higher (a factor of 9.5) than the standard PN abundance set.

The average temperatures, $T_{\mathrm{A}}$, as a function of time for all the models are plotted in Figure 7 As expected, the average temperature is always close to the peak temperature of the emission coefficient. Moreover, the average temperature values are in agreement with those derived from observations of hot bubbles in PNe, which generally fall in the range $(1-3) \times 10^{6} \mathrm{~K}$ (see Ruiz et al. 2013). In general, we find that without conduction (Fig. 7- open symbols), the average temperature rises and then slowly decreases for all stellar models. The 2.0-0.633 model without conduction reaches the highest average plasma temperature $T_{\mathrm{A}} \sim 4 \times 10^{6} \mathrm{~K}$ of all the models studied.

When thermal conduction is included, the average plasma temperature rises steadily throughout the evolution, until a constant value of $\log \left(T_{\mathrm{A}}\right) \sim 6.2$ (i.e., $T_{\mathrm{A}} \sim$ $\left.1.6 \times 10^{6} \mathrm{~K}\right)$ is reached, except for the highest mass model (2.5-0677). This high-mass model evolves so quickly that the average plasma temperature behaves in a similar way to its counterpart without conduction.

The lowest mass model, 1.0-0.569, has an average temperature that increases very slowly with time. This is because the CSPN fast wind velocity in this model increases slowly as a function of time and even after 10,000 yrs has only reached $\sim 600 \mathrm{~km} \mathrm{~s}^{-1}$ (see Fig. A1). Consequently, even at the latest times in the simulation there is little gas at or above the peak temperature of the emission coefficent (see Fig. 5) and the average temperature derived from this model climbs slowly.

The result that models with thermal conduction show very uniform values of the mean temperature, both as a function of time and between models for different initial stellar masses suggests that the properties of the conduction layer are essentially independent of the stellar parameters in our models. The variation and higher mean temperatures of the models without conduction can be explained by looking at the DEM plots (Fig. 5). The important factor is the relative value of the DEM at higher temperatures to the DEM around $\log (T)=6$, which indicates the importance of the hotter gas to the mean temperature. At early times, the increase in stellar wind velocity, relatively high mass-loss rates and small volume of the hot bubble mean that high temperature gas is a major contributor to the DEM in the models without conduction, while at later times, although the maximum temperature is higher, the contribution to the DEM in these models is relatively smaller due to lower densities and geometrical dilution and because the turbulent mixing layer around the filaments consists mainly of lower temperature gas.

It is also interesting to compare the average plasma temperature $\left(T_{\mathrm{A}}\right)$ as a function of other parameters, e.g., hot bubble radius, stellar effective temperature, and terminal wind velocity. Figure 8 (left-hand panel) shows that small bubbles $\left(R_{\mathrm{HB}}<0.05 \mathrm{pc}\right)$ can have a variety of mean plasma temperatures, $0.4 \times 10^{6}<T_{\mathrm{A}}<2.6 \times 10^{6} \mathrm{~K}$, depending on the stellar mass model. On the other hand, large bubbles $\left(R_{\mathrm{HB}}>0.2 \mathrm{pc}\right)$ all have temperatures in the narrow range $1.5 \times 10^{6}<T_{\mathrm{A}}<1.8 \times 10^{6} \mathrm{~K}$ (see figure 6 in Ruiz et al. 2013). In our simulations, average temperatures above $T_{\mathrm{A}}>2.5 \times 10^{6} \mathrm{~K}$ are only found for models without conduction and intermediate hot bubble radii. Furthermore, the comparisons with effective temperature and terminal wind velocity (Fig. 8 central and right-hand panels) show that the largest values of the average temperature in the models without conduction are not associated with either the maximum stellar effective temperature or the peak wind power (see also Fig. A1).

We include in Fig. 8 the corresponding derived characteristic temperatures for the X-ray observations of objects listed in Ruiz et al. (2013) (numbered star symbols; see Table 1 and references therein). One of these points (numbered 1) corresponds to the $\mathrm{PN} \mathrm{BD}+30^{\circ} 3639$, which is included as it correponds to the best-quality observa- 

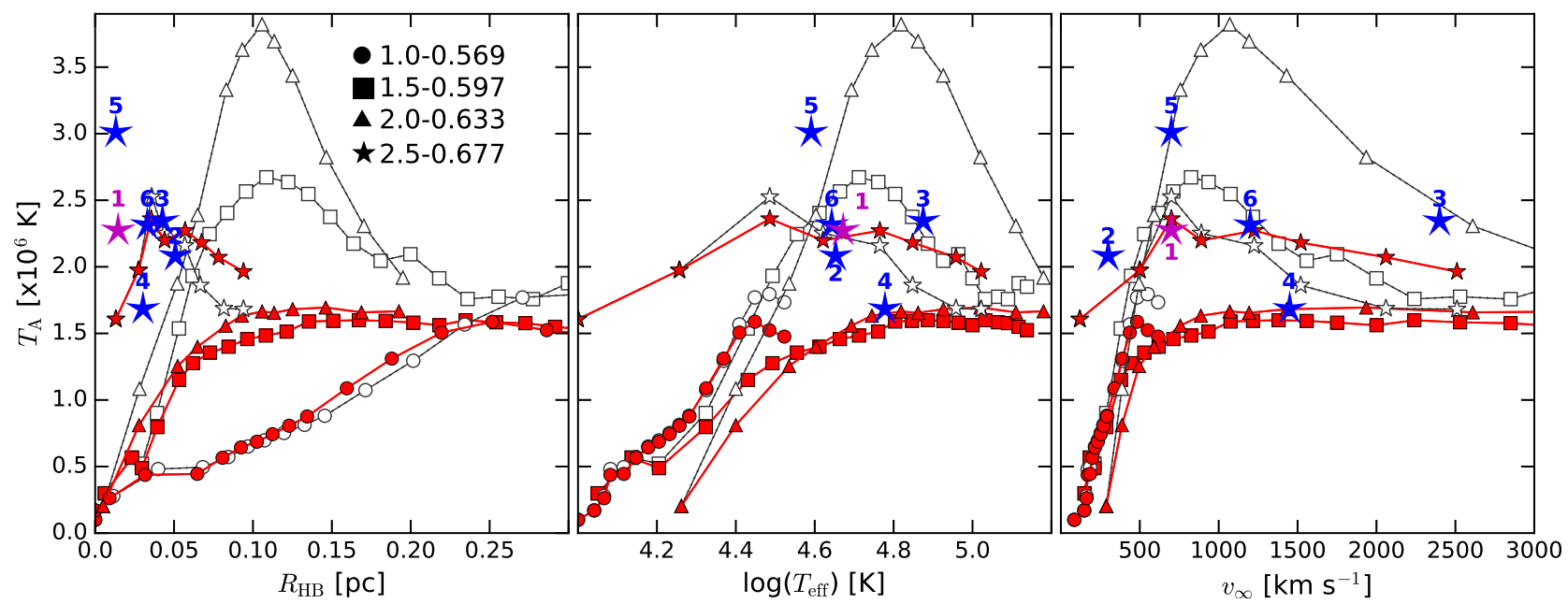

Figure 8. Average plasma temperature as a function of hot bubble radius (left panel), stellar effective temperature (middle panel) and stellar wind velocity (right panel) for different models. Filled and open symbols are for the models with and without thermal conduction, respectively. The stars, labeled from 1 to 6 , correspond to observations of BD+30 3639 , NGC 2392 , NGC 3242, NGC 6543, IC 418, and NGC 6826 respectively (see Table 1).

Table 1. X-ray properties of selected PNe

\begin{tabular}{|c|c|c|c|c|c|c|c|c|c|c|}
\hline Label & Object & $\begin{array}{c}\text { Hot Bubble } \\
\text { Radius } \\
\text { (arcsec) }\end{array}$ & $\begin{array}{l}\text { distance } \\
\text { (kpc) }\end{array}$ & $\begin{array}{c}L_{\mathrm{X}}^{\mathrm{a}} \\
(\mathrm{cgs})\end{array}$ & $\begin{array}{c}F_{\mathrm{X}}^{\mathrm{a}} \\
(\mathrm{cgs})\end{array}$ & $\begin{array}{c}S_{\mathrm{X}}^{\mathrm{a}} \\
(\mathrm{cgs})\end{array}$ & $\begin{array}{l}k T_{\mathrm{X}}^{\mathrm{a}} \\
(\mathrm{keV})\end{array}$ & $\begin{array}{l}T_{\mathrm{eff}}^{\mathrm{b}} \\
(\mathrm{kK})\end{array}$ & $\begin{array}{c}v_{\infty}^{\mathrm{b}} \\
\left(\mathrm{km} \mathrm{s}^{-1}\right)\end{array}$ & References ${ }^{\mathrm{e}}$ \\
\hline 1 & $\mathrm{BD}+30^{\circ} 3639^{\mathrm{c}}$ & 2.5 & 1.20 & $7.4 \times 10^{32}$ & $4.3 \times 10^{-12}$ & $2.2 \times 10^{-13}$ & 0.20 & 47 & 700 & $(1,2,3)$ \\
\hline 2 & NGC 2392 & 8.2 & 1.28 & $1.8 \times 10^{31}$ & $9.2 \times 10^{-14}$ & $4.3 \times 10^{-16}$ & 0.18 & 45 & 300 & $(4,5)$ \\
\hline 3 & NGC 3242 & 8.8 & 1.00 & $7.3 \times 10^{30}$ & $6.1 \times 10^{-14}$ & $2.5 \times 10^{-16}$ & 0.20 & 75 & 2400 & $(6,7,8)$ \\
\hline 4 & NGC 6543 & 6.2 & 1.00 & $1.0 \times 10^{32}$ & $8.0 \times 10^{-13}$ & $6.5 \times 10^{-15}$ & 0.15 & 50 & 1450 & $(5,7,9)$ \\
\hline 5 & $\mathrm{IC} 418^{\mathrm{d}}$ & 2.2 & 1.20 & $8.4 \times 10^{29}$ & $4.9 \times 10^{-15}$ & $3.1 \times 10^{-16}$ & 0.26 & 39 & 700 & $(4,6,7)$ \\
\hline 6 & NGC $6826^{\mathrm{d}}$ & 5.3 & 1.30 & $2.0 \times 10^{30}$ & $9.9 \times 10^{-15}$ & $1.1 \times 10^{-16}$ & 0.20 & 44 & 1200 & $(4,6,7)$ \\
\hline
\end{tabular}

${ }^{a} L_{\mathrm{X}}, F_{\mathrm{X}}, S_{\mathrm{X}}$, and $k T_{\mathrm{X}}$ are the X-ray luminosity, unabsorbed flux, surface brightness, and plasma temperature as obtained from observations. $L_{\mathrm{X}}, F_{\mathrm{X}}, S_{\mathrm{X}}$ are presented in cgs units, that is, $\left(\mathrm{erg} \mathrm{s}^{-1}\right),\left(\mathrm{erg} \mathrm{s}^{-1} \mathrm{~cm}^{-2}\right)$, and $\left(\mathrm{erg} \mathrm{s}^{-1} \mathrm{~cm}^{-2} \operatorname{arcsec}^{-2}\right)$, respectively.

${ }^{\mathrm{b}} T_{\text {eff }}$ and $v_{\infty}$ are the stellar effective temperature and terminal wind velocity of the CSPN.

${ }^{\mathrm{c}} \mathrm{PNe}$ with [WC]-type CSPN.

dPNe with no strict fit to the data (see Ruiz et al. 2013)

e'References: (1) Yu et al. (2009), (2) Li et al. (2002), (3) Marcolino et al. (2007), (4) Ruiz et al. (2013), (5) Herald \& Bianchi (2011), (6) Ruiz et al. (2011), (7) Pauldrach et al. (2004), (8) Frew (2008) and (9) Chu et al. (2001).

tion among all reported $\mathrm{PNe}$ to date even though it has a [WC]-type CSPN (e.g., Yu et al. 2009). Other PNe with [WR]-type CSPN have been left outside?. We remark that our estimated surface brightness for IC 418, NGC 2392, and NGC 6826 differ from those listed by Ruiz et al. (2013) for a factor of $\sim 4$. The authors used erroneously the diame-

${ }^{3}$ Note that we do not include in our table NGC7009 and NGC 7027. The analysis of the X-ray emission reported so far for NGC 7009 was performed with XMM-Newton observations (Guerrero et al. 2002) and did not have the spatial resolution to separate the CSPN emission from the diffuse X-ray emission (see figures 3 and 4 in Kastner et al. 2012). On the other hand, we do not use the derived X-ray properties of NGC 7027 since extinction within the nebula precludes a clear analysis of the hot bubble. ters instead of the radii of the $\mathrm{PNe}$ to calculate the surface brightness (M.A. Guerrero private communication). It is also important to bear in mind that the $\mathrm{X}$-ray properties of IC 418 and NGC 6826 were obtained without performing a rigorous fit to the data. Other X-ray properties of the $\mathrm{PNe}$ listed in our table have been updated.

Figure 8 (centre panel) shows that our models with and without conduction span the range of mean plasma temperatures derived from observations, when plotted against the stellar effective temperature or stellar wind velocity. On the other hand, the observational points appear to favour the highest mass models when the size of the hot bubble is taken into consideration, since these correspond to the smallest bubbles. We note that all of the brightest ob- 
served $\mathrm{PNe}$ showing diffuse $\mathrm{X}$-rays have bubbles with radii $R_{\mathrm{HB}}<0.05$ pc.

In Appendix B we show the average temperatures of the 1D simulations of the 1.5-0.597 models with and without conduction both as a function of time and as a function of stellar effective temperature (see Fig. B33). The average temperatures of the $1 \mathrm{D}$ simulations without conduction climb to $T_{\mathrm{A}} \sim 10^{7} \mathrm{~K}$, very different to the $2 \mathrm{D}$ case, because there is very little gas around the temperature corresponding to the peak in the emission coefficient (see Fig. B2). The 1D simulations with thermal conduction reach slightly higher average temperatures than their $2 \mathrm{D}$ counterparts, because the thermal conduction layer is not so extensive in the $1 \mathrm{D}$ models due to the absence of corrugations.

\subsection{Spectra}

The synthetic X-ray emission for each model at each time is computed using the extensively tested CHIANTI database and software package (Version 7.1.3; Dere et al. 1997, 2009; Landi et al. 2013). The calculation performed by CHIANTI includes the contributions due to lines and to free-free, freebound, and two-photon continua. We used the updated default ionization equilibrium ion fractions included in the latest version of CHIANT (see Landi et al. 2013, and references therein). The abundances used to compute the spectra are those defined for a standard PN composition in Cloudy version 13.0 (Ferland et al. 2013), for consistency with Paper I. A spectrum is generated for each of the temperature bins and weighted by the appropriate DEM value. The complete spectrum is simply the sum of all these individual contributions.

All synthetic spectra are computed in the $0.3-2.0 \mathrm{keV}$ energy range, corresponding to $6-40 \AA$ in the wavelength range, with a spectral bin of $0.01 \AA$ and the spectral line FWHM is assumed to be $0.1 \AA$.

\subsection{Absorption}

To facilitate direct comparison with observations, the intrinsic spectra produced with the CHIANTI software should be corrected for absorption by neutral material along the line of sight. Extinction is particularly important for energies $<0.5 \mathrm{keV}$ and can reduce the spectral intensity by an order of magnitude or more.

We use the the photoelectric absorption cross sections from Balucińska-Church \& McCammon (1992) and standard ISM abundances. We consider two values for the atomic hydrogen column density: a moderate value $N_{\mathrm{H}}=$ $8 \times 10^{20} \mathrm{~cm}^{-2}$, and a high value $N_{\mathrm{H}}=5 \times 10^{21} \mathrm{~cm}^{-2}$. These values are within the range derived from observations (e.g., Ruiz et al. 2013). Since the spectra of PNe are generally quite soft, extinction plays an important rôle in the shape of the spectrum at lower energies. Even so, there are some PNe for which an important count rate has been measured at low energies (e.g., NGC 6543 and $\mathrm{BD}+30^{\circ} 3639$; Kastner et al. 2000; Chu et al. 2001; Yu et al. 2009).

Finally, the absorbed spectrum should be convolved with the instrumental response matrices. Convolution with

4 see: http://www.chiantidatabase.org/cug.pdf

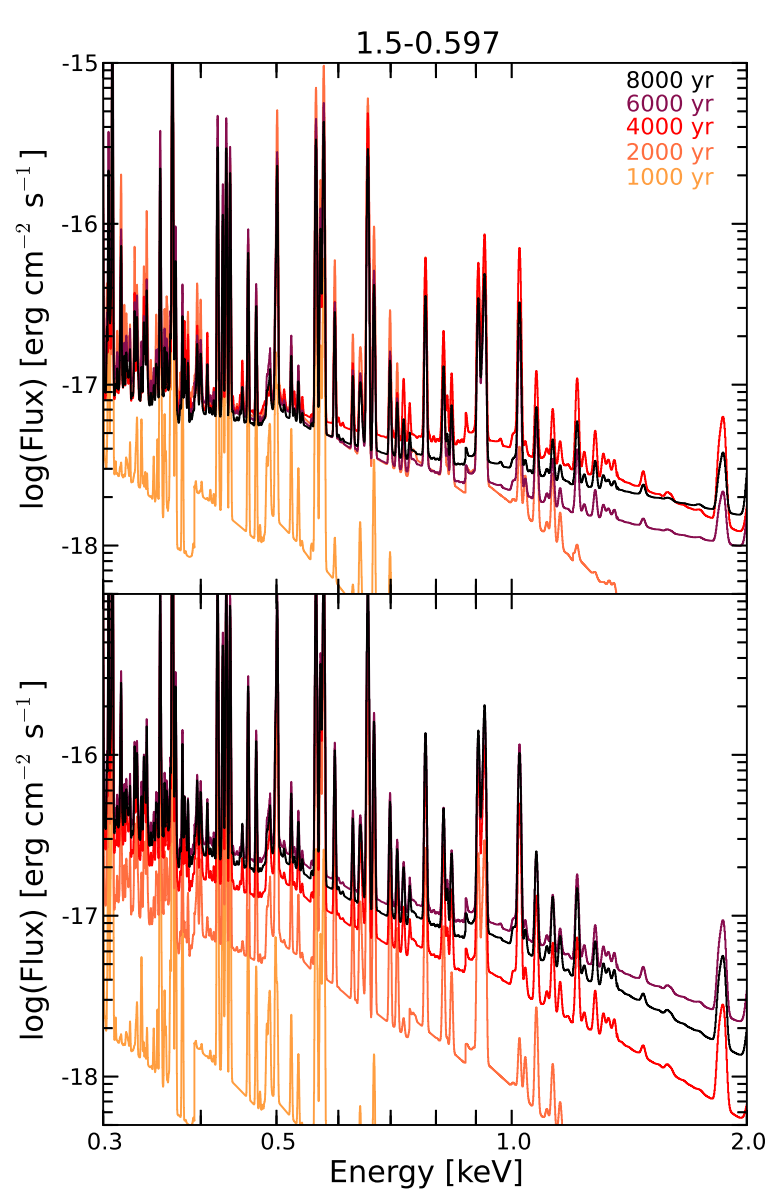

Figure 9. Time evolution of the spectra in the $0.3-2.0 \mathrm{keV}$ energy range for the 1.5-0.597 model at different times after the onset of the post-AGB phase. Top: Without thermal conduction. Bottom: With thermal conduction. Colours represent different times.

the XMM-Newton EPIC-pn and Chandra ACIS instrumental responses and effective areas can be done within the CHIANTI software after the absorption has been applied.

\section{RESULTS}

\subsection{Synthetic X-ray spectra}

As an example of the resultant synthetic X-ray spectra obtained with CHIANTI using the procedure described in 2.3 Figure 9 shows total intrinsic (i.e., non-absorbed) spectra for the 1.5-0.597 model with and without thermal conduction at different times after the onset of the post-AGB phase. The spectra are plotted in the energy range $0.3-2.0 \mathrm{keV}$ as this corresponds to the range generally presented in observational studies of the diffuse X-rays from $\mathrm{PNe}$ (e.g., Ruiz et al. 2013, and references therein). All spectra show a great number of spectral lines but there are noticeable differences between models with and without thermal conduction.

The case without thermal conduction (Figure 9] top 


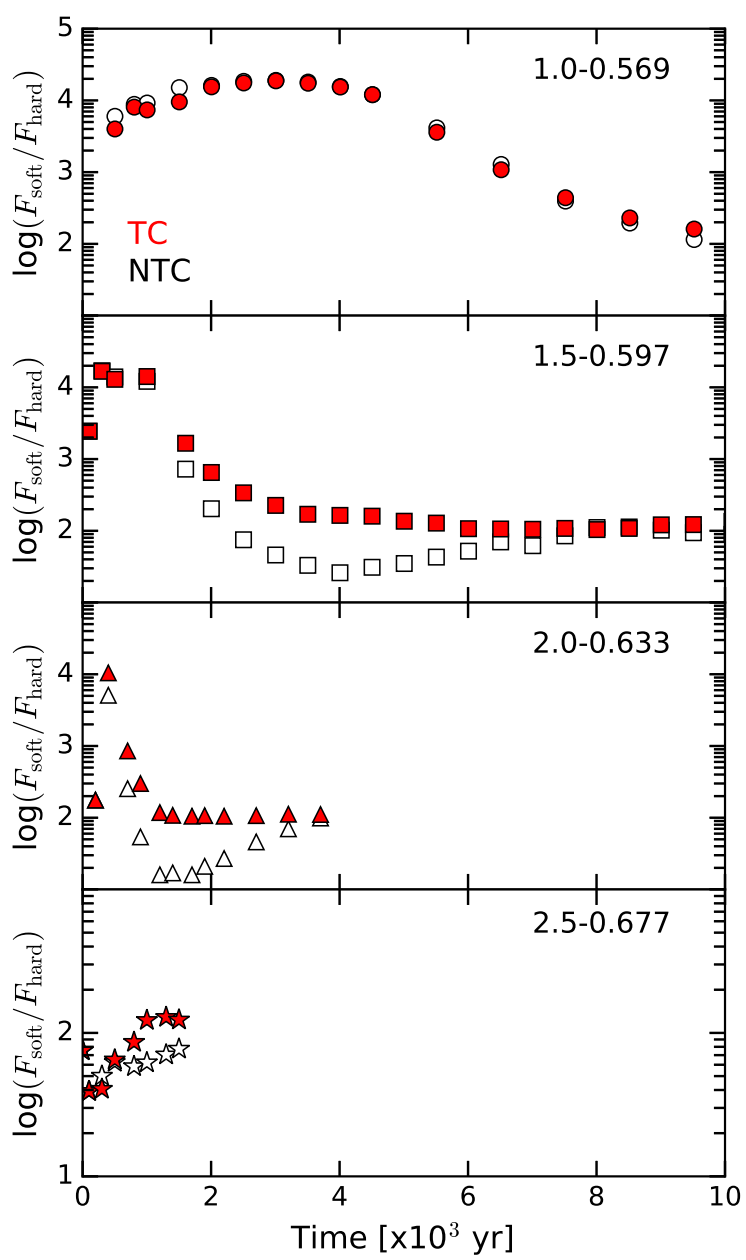

Figure 10. Variation of the ratio of soft $(0.3-1.0 \mathrm{keV})$ to hard (1.0-2.0 keV) X-ray fluxes with time for all models. Filled and open symbols represent results with (TC) and without thermal conduction (NTC), respectively.

panel) shows an evolution in the slope of the continuum with time. This follows from the time evolution of the DEM distributions seen in Fig. 5 and the average temperature seen in Fig. 7. We have seen how, for the 1.5-0.597 model, the average temperature increases gradually to reach a maximum around 4000 yrs after the end of the AGB stage and then decreases. The changing average temperature is responsible for the changing slope of the continuum. Furthermore, from Fig. 5 we see that the general trend is for the values of the DEM distribution to increase with time. This is responsible for the general increase in the fluxes as time progresses.

On the other hand, the average temperature for the 1.50.597 model with conduction remains constant after 3000 yrs (see Fig. 77). For this reason, the slope of the continuum does not change in Fig. 9] The fluxes increase because the DEM values increase as time advances.

The results for the $1 \mathrm{D}$ simulations of the $1.5-0.597$ model are given in Appendix B. Although the simulated spectra for the $1 \mathrm{D}$ cases with thermal conduction show similar behaviour to their 2D counterparts, the results for the cases without conduction are very different. Due to the very high average temperature $\left(T_{\mathrm{A}} \sim 10^{7} \mathrm{~K}\right)$, the spectral shape of the $1 \mathrm{D}$ results without conduction is essentially flat in the 0.3-2.0 keV energy range.

Another way of looking at the evolution of the spectral shape is to consider the hardness ratio. This is the ratio of the soft-band (0.3-1.0 keV) luminosity to the hard-band $(1.0-2.0 \mathrm{keV})$ luminosity. In Figure 10 we show the variation of the hardness ratio as a function of time for all models. The hardness ratio reflects the behaviour of the average temperature, $T_{\mathrm{A}}$ (see Fig. 7): for lower average temperatures the spectrum will be softer. The models with conduction generally have softer spectra than those without (except for the highest mass model 2.5-0.677), which is consistent with their average temperatures being lower. The hardness ratios of the models with conduction show less variation than those without conduction because the conduction layer dominates the $\mathrm{X}$-ray emissivity and is weighted toward lower temperatures (see Fig. 5). For the models without conduction, the spectra are hardest when the average temperature is highest, which occurs while the hot bubble is still reasonably compact and the DEM distribution has an important contribution from high temperature gas.

At early times, the soft band dominates because the plasma average temperatures are low as a consequence of the low stellar wind velocity. As time goes on, the wind velocity, and hence the plasma average temperature, increases and the contribution to the hard X-ray band becomes more important. The models without conduction reach higher average temperatures than the models with conduction, and this is evident by the low values of the hardness ratio $F_{\text {soft }} / F_{\text {hard }}$, which correspond exactly to the peak in $T_{\mathrm{A}}$ seen in Figure 7 On the other hand, for the most massive model 2.5-0.677, the stellar wind evolves so quickly that the properties of the mixing layer dominate the spectra from early times.

\subsection{Luminosities}

The X-ray luminosities, $L_{\mathrm{X}}$, in the $0.3-2.0 \mathrm{keV}$ energy band are found by integrating the synthetic spectra over this energy range. In Figure 11 top left panel we show the time evolution of the X-ray luminosity for all models used in the present paper. The first thing to notice is that all models result in a wide range of $\mathrm{X}$-ray luminosities within the $\mathrm{X}$ ray luminosity range reported by observations (see Table1). As anticipated, models with thermal conduction have higher X-ray luminosities (by up to an order of magnitude in the case of the two most massive models) compared to the corresponding models without thermal conduction.

In all our models, the luminosity behaviour seen in Figure 11 can be understood with reference to the DEM histograms shown in Figure 5 In particular, the DEM value around $T \sim T_{\mathrm{A}} \sim 1.5 \times 10^{6} \mathrm{~K}$ shows that for the lower mass models 1.0-0.569 and 1.5-0.597, the luminosity rises at first then stays more-or-less constant as the hot bubble continues to expand for the cases both with and without conduction. For the 2.0-0.633 models DEM value at the average plasma temperature rises continually for the duration of the simulation. The highest mass models 2.5-0.677 have high luminosity even from early times. 

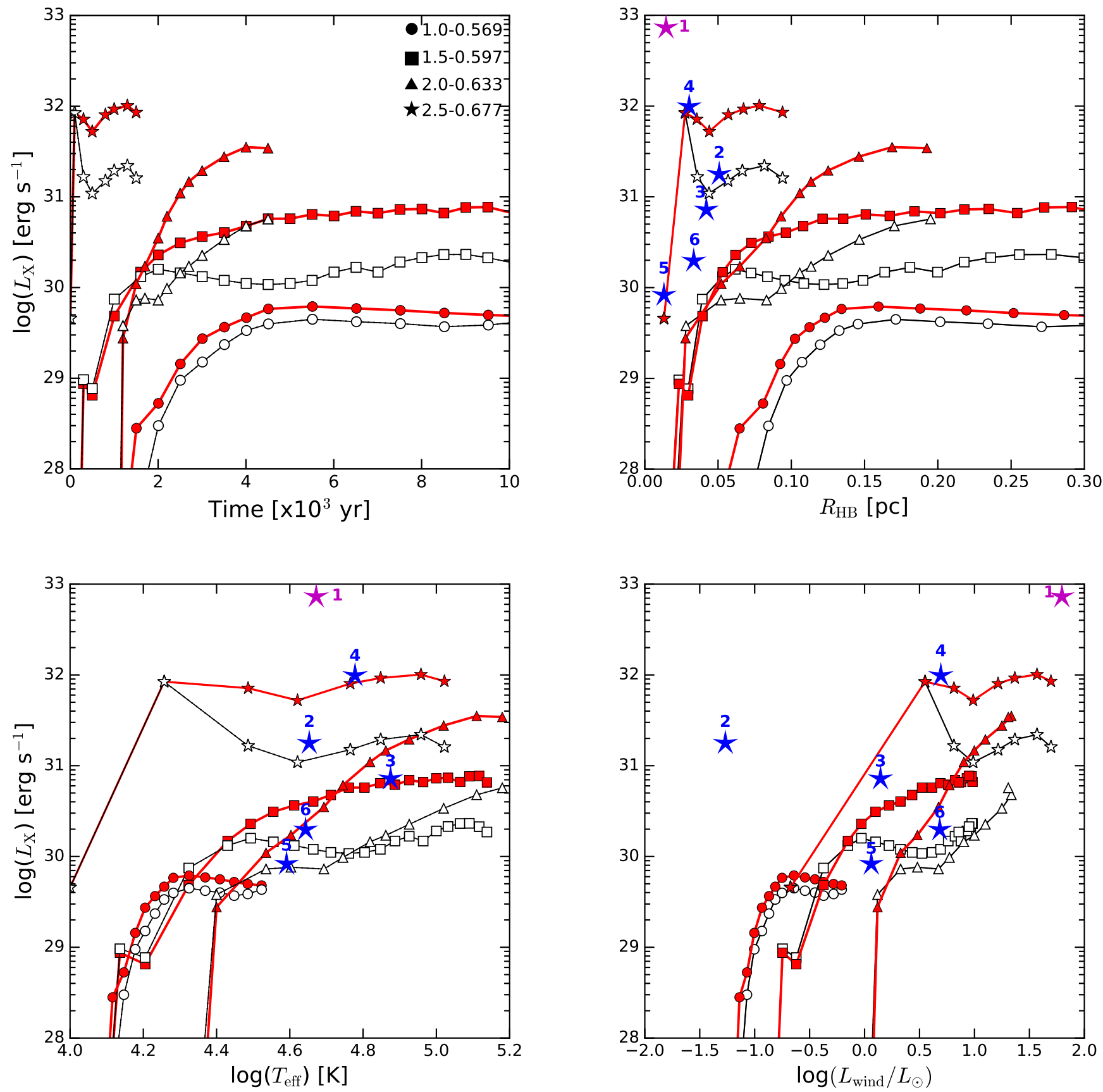

Figure 11. Evolution of the X-ray luminosity in the $0.3-2.0 \mathrm{keV}$ energy range as a function of time (upper left panel), hot bubble radius (upper right panel), stellar effective temperature (lower left panel), and stellar wind mechanical luminosity (lower right panel). Filled and open symbols represent models with and without thermal conduction, respectively. Stars, labeled from 1 to 6 , correspond to observations of $\mathrm{BD}+30^{\circ} 3639$, NGC 2392, NGC 3242, NGC 6543, IC 418, and NGC 6826 respectively (see Table 1).

The luminosity as a function of hot bubble radius (Fig. 11] upper right panel) shows that the less massive models 1.0-0.560 and 1.5-0.597 show little variation in their luminosities over the radius range $0.05<R_{\mathrm{HB}}<0.2$ pc. Only the most massive model (2.5-0.677) is capable of producing very small $\left(R_{\mathrm{HB}}<0.05 \mathrm{pc}\right)$, highly X-ray luminous bubbles. Unfortunately, we cannot follow the evolution of these models at larger sizes. This figure also plots the obser- vationally derived luminosities and radii for the six objects listed in Table 1 Interestingly, all these objects have very small diffuse X-ray bubbles.

As a function of the CSPN effective temperature (Fig. 11, lower left panel), all models show a steady increase in X-ray luminosity with $T_{\text {eff }}$. With the exception of Object $1\left(\mathrm{BD}+30^{\circ} 3639\right)$, our set of models encompasses the full range of observed luminosities and corresponding effective 

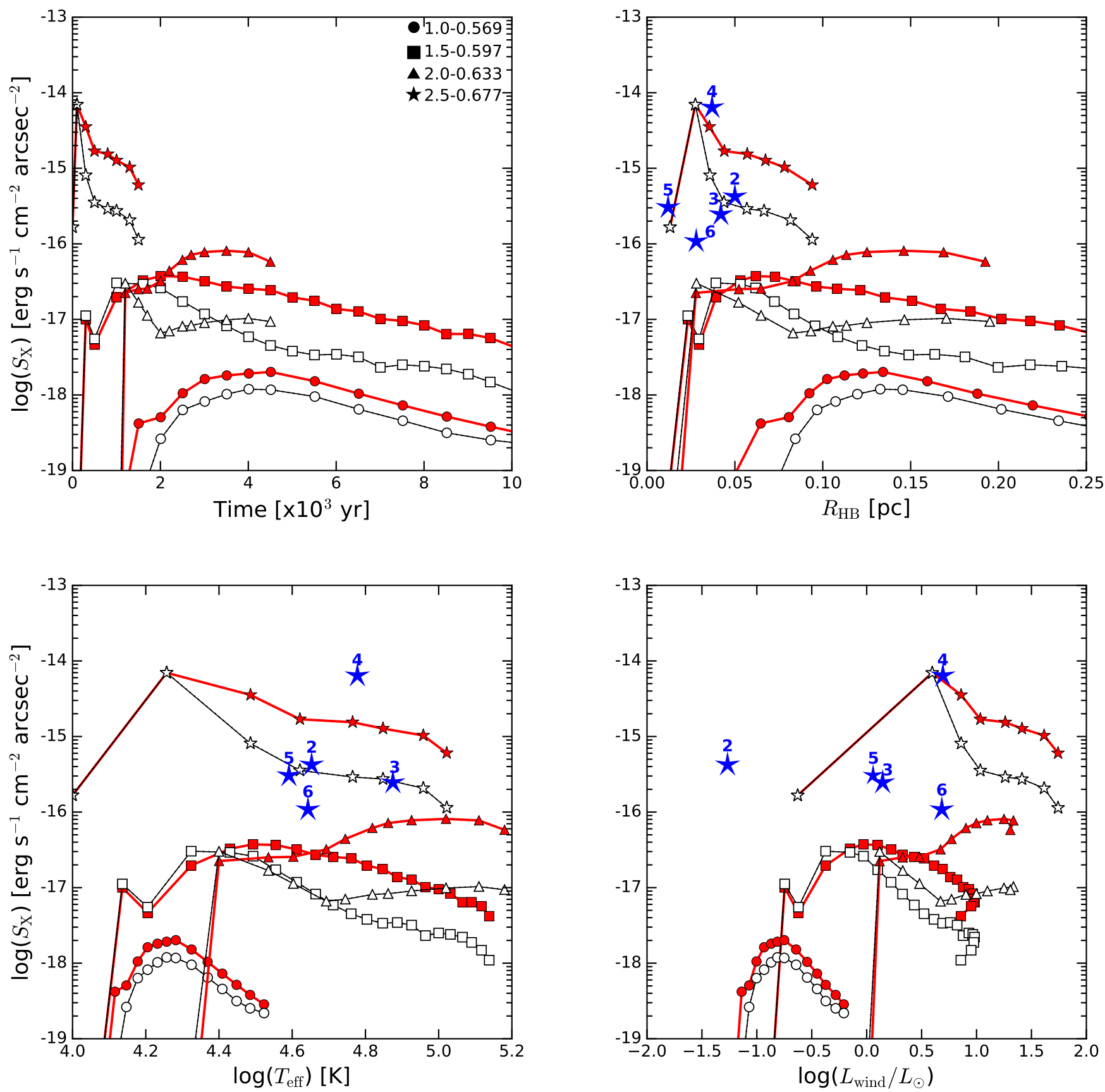

Figure 12. Evolution of the X-ray averaged surface brightness in the $0.3-2.0 \mathrm{keV}$ energy range for all models as a function of time (upper left panel), hot bubble radius (upper right panel), stellar effective temperature (lower left panel), and stellar wind mechanical luminosity (lower right panel). Filled and open symbols represent models with and without thermal conduction, respectively. Stars, labeled from 2 to 6, correspond to observations of NGC 2392, NGC 3242, NGC 6543, IC 418, and NGC 6826 respectively (see Table 1).

temperatures listed in Table 1. Even models without thermal conduction are capable of producing the luminosities of several observed objects. However, other derived parameters, particularly the radius of the X-ray emitting bubble, should be taken into account when determining the best-fit model. Our models do not proceed beyond the maximum $T_{\text {eff }}$ of each CSPN (see Fig. A1) for the technical reasons described earlier and so none of our models correspond to objects whose CSPN is descending the white dwarf cooling track. The 1.0-0.569 model is included for completeness, even though it is unlikely to correspond to any object detected with current instruments since the predicted diffuse X-ray luminosities are low $\left(L_{\mathrm{X}}<10^{30} \mathrm{erg} \mathrm{s}^{-1}\right)$.

Finally, Fig. 11 lower-right panel shows the X-ray luminosity as a function of stellar wind mechanical luminosity for all the models. In this figure it should be borne in 
mind that the observational points have poorly determined mass-loss rates and hence the corresponding wind mechanical luminosities are also uncertain. In particular, Object 2 (NGC 2392) has a very weak stellar wind whose velocity is difficult to determine but which has been estimated as $v_{\infty} \sim 300 \mathrm{~km} \mathrm{~s}^{-1}$ (Herald \& Bianchi 2011).

Since we know the radius of the hot bubble as a function of time for each model (Fig. 4), we can also calculate the expected averaged surface brightness. The surface brightness as a function of time, hot bubble radius, CSPN effective temperature, and stellar wind mechanical luminosity are plotted in Figure 12. In comparison to the equivalent luminosity curves (see Fig. 11), the surface brightness shows more constant values in time periods where the luminosity is increasing. This is because the increase in the bubble size offsets the increase in luminosity. The values we obtain for the (unabsorbed) surface brightness are within the range reported by the objects in Table 1 but only for the most massive model 2.5-0.677. Only this model can produce small, bright X-ray bubbles. All our other models produce low surfacebrightness objects. More extensive observational campaigns, such as CHANPlaNS and follow-up observations, should improve the detections of low surface-brightness objects.

Finally, we plot the X-ray luminosity in the energy range $0.3-2.0 \mathrm{keV}$ as a fraction of the stellar wind mechanical luminosity (see Fig. 13) against time and also as a function of CSPN effective temperature. Generally, less than $1 \%$ of the stellar wind mechanical luminosity is converted into Xray luminosity This is consistent with the results presented by Steffen et al. (2008) for their efficient conduction models, and with the handful of observations that support their models. Initially the models with and without conduction convert the similar fractions of stellar wind mechanical luminosity to soft X-ray luminosity. However, as time proceeds, the models with conduction convert a factor of up to an order of magnitude more stellar wind luminosity to X-rays. This is because the $\mathrm{X}$-rays generally come from the vicinity of the mixing layer, where conditions are more uniform and to some extent immune from rapid changes in the stellar wind parameters.

\subsection{Contribution of the shocked fast wind}

In Section 2 we described the use of an advected scalar $s$ in our numerical simulations (see also Paper I). As shown before, this scalar is useful for delimiting the different fluid contributions to the bubble structure (see Fig. 3). With this parameter, we can separate the contributions of the shocked fast stellar wind and the evaporated/mixed nebular materia 5 to the total X-ray luminosity. In the mixing region, the scalar takes intermediate values between $s=0$ (fast wind) and $s=1$ (nebular material). For simplicity, we use $s=0.5$ as the value that distinguishes the two fluids, i.e. $0<s<0.5$ is dominated by fast wind material, while $0.5<s<1$ is dominated by nebular material.

As described in Section 2.1 the first step in calculating the contribution of the shocked fast stellar wind to the total

\footnotetext{
5 The nebular material surrounded the star at the onset of the fast wind stage and was expelled from the stellar envelope during the previous AGB stage in a dense, slow wind.
}
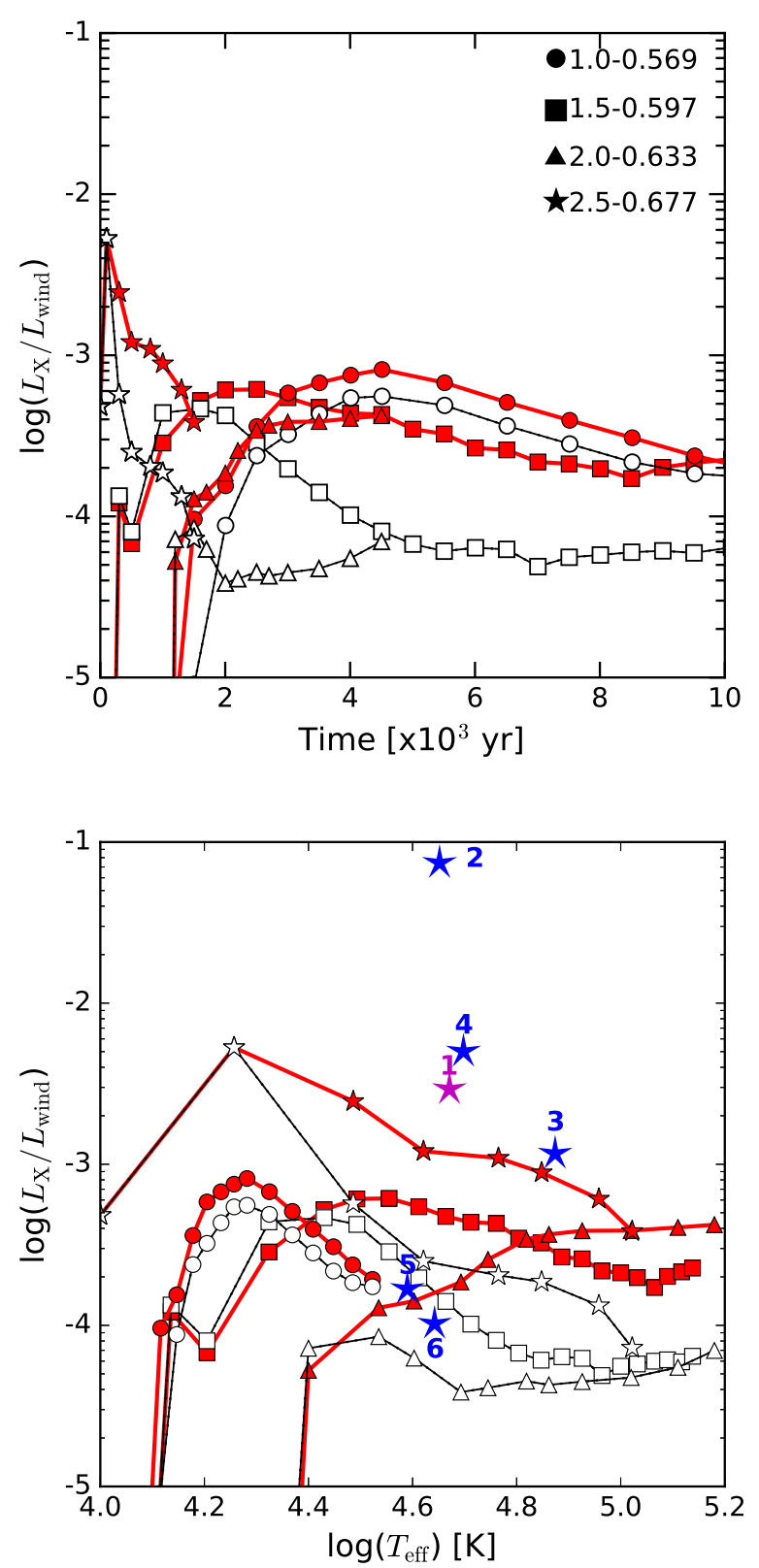

Figure 13. Evolution of the X-ray luminosity as a fraction of the CSPN wind mechanical luminosity for all models. Top panel: $L_{\mathrm{X}} / L_{\text {wind }}$ as a function of time. Bottom panel: $L_{\mathrm{X}} / L_{\text {wind }}$ as a function of central star effective temperature. Filled and open symbols represent models with and without thermal conduction, respectively.

X-ray luminosity is to compute the DEM for material corresponding to $0<s<0.5$. The resultant DEMs, together with the CHIANTI software, were used to compute spectra, and hence the X-ray luminosities in the 0.3-2.0 keV energy range, $L_{\mathrm{X}, 0-0.5}$, corresponding to the shocked fast stellar wind gas (see Sections 2.3 and 3.2 ).

Figure 14 shows the time evolution of the DEMs computed for the scalar range $(0<s<0.5)$ together with those calculated for the total scalar range $(0<s<1)$ for the 1.5-0.597 model with and without thermal conduction. At 


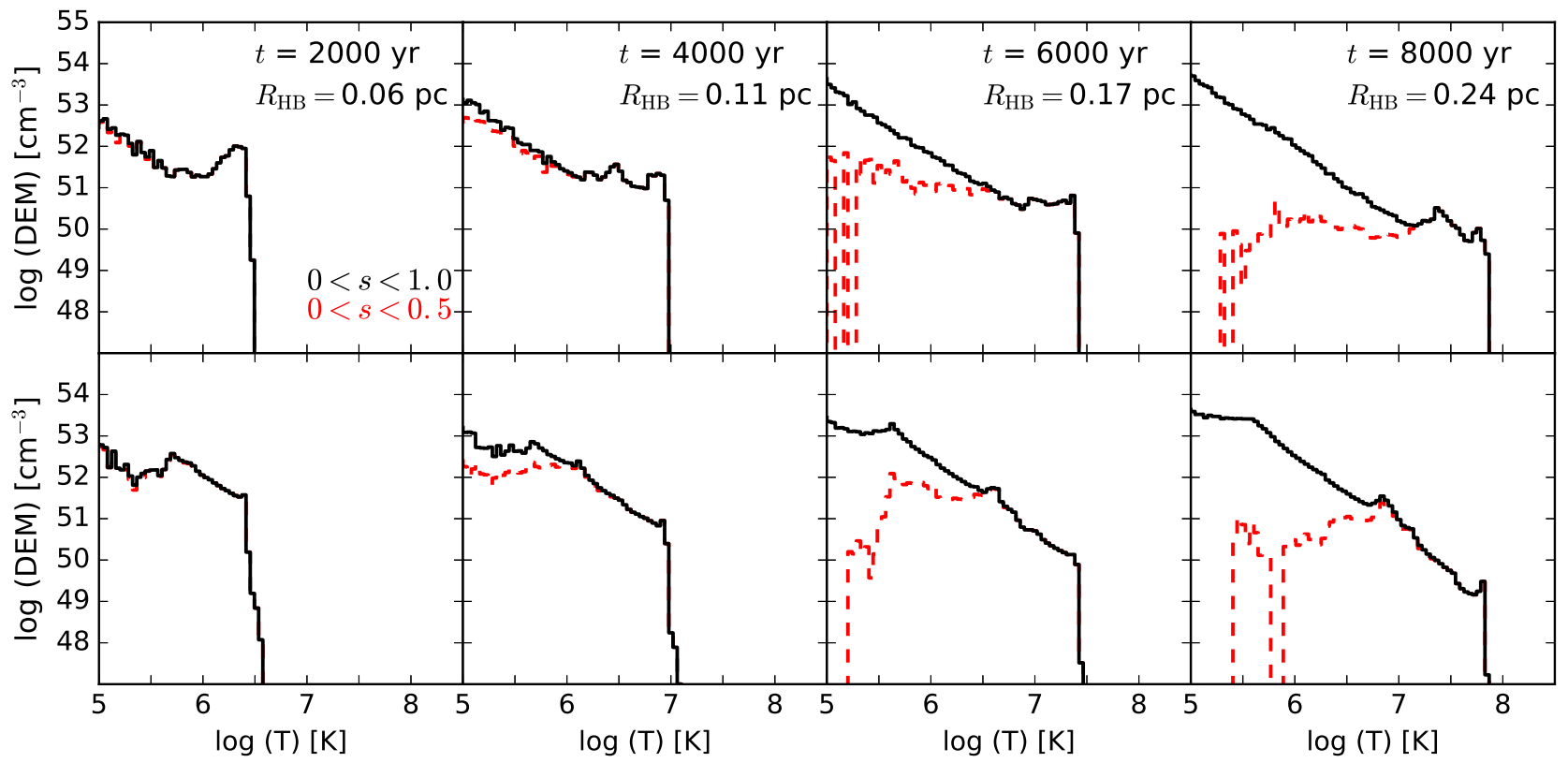

Figure 14. Evolution of DEM for two different ranges of the scalar values for the 1.5-0.597 model. Solid (black) lines correspond to DEM computed over the complete scalar range $(0<s<1)$ while the dashed (red) lines are the results of taking only the shocked fast stellar wind contribution $(0<s<0.5)$. Upper and lower panels show the results without and with thermal conduction, respectively. Times and the corresponding radius of the hot bubble are marked on each column.

the earliest times $(t<2000$ yrs), the shocked fast wind is the dominant contributor to the DEM at all temperatures $T>10^{5} \mathrm{~K}$. The photoionized nebular gas has temperatures $T \sim 10^{4} \mathrm{~K}$.

As time progresses, the contribution of the nebular gas to the X-ray luminosity increases due to the expansion of the shell and the incorporation of new material into the mixing and conduction layers. In contrast, the contribution of the hot shocked wind remains fairly constant, since the wind velocity increases with time and so shocks to higher and higher temperatures, which do not contribute to the diffuse X-ray luminosity. At late times $(t>6000$ yrs) the nebular material dominates the DEM distributions in both cases, except at the very highest temperatures $(\log T>7)$; it is the main contributor in the turbulent mixing layer for the models without conduction and it is the main contributor to the conduction layer in the other set of models.

Figure 15 shows the ratio of the X-ray luminosity of the shocked fast stellar wind material to the total X-ray luminosity (in the $0.3-2.0 \mathrm{keV}$ energy range: $L_{\mathrm{X}, 0-0.5} / L_{\mathrm{X}}$ ) as a function of time for all stellar models. This figure demonstrates that at early times the shocked stellar wind gas dominates the total X-ray luminosity for all cases. This is because neither the turbulent mixing regions nor the conduction layers nor any other possible mechanism for heating the nebular material have had enough time to develop. As time progresses, the $L_{\mathrm{X}, 0-0.5} / L_{\mathrm{X}}$ ratio decreases because while the fast stellar wind contribution to the diffuse X-ray luminosity in the $0.3-2.0 \mathrm{keV}$ energy band remains roughly constant, the total diffuse X-ray luminosity increases due to the incorporation of new material into the mixing layers that accompanies the expansion of the hot bubble. The small dif- ferences in the $L_{\mathrm{X}, 0-0.5} / L_{\mathrm{X}}$ ratio seen between models with and without conduction for a given stellar mass are due to the X-ray luminosity difference (typically up to a factor of 4) between both sequences, as seen in Figure 11 These differences tend to be larger with increasing stellar mass.

Finally, we remark that the luminosity ratios for the 1.0-0.569 models remain almost constant for the cases with and without conduction, thus demonstrating that all X-ray properties dervied from these models are due almost entirely to the shocked fast stellar wind material.

\section{COMPARISON WITH OBSERVATIONS}

\subsection{Correction for absorption and convolution with instrumental response matrices}

The intrinsic spectra shown in Figure 9 need to be corrected for interstellar absorption and convolved with the instrumental response matrices before they can be compared with observations. As an illustration, Figure [16] shows the effects of absorption on the intrinsic spectrum of the 1.50.597 model without thermal conduction at $8000 \mathrm{yr}$. Results are shown for both moderate $\left(N_{\mathrm{H}}=8 \times 10^{20} \mathrm{~cm}^{-2}\right)$ and high $\left(N_{\mathrm{H}}=5 \times 10^{21} \mathrm{~cm}^{-2}\right)$ neutral hydrogen column densities. The principal emission lines are identified in this figure, showing clearly how carbon and nitrogen dominate the lowenergy spectrum for the abundances used in this work, while oxygen and neon emission lines are important at high energies. Figure 16 shows how the most intense X-ray-emitting part of the spectrum is severely affected by extinction. For example, the emission below $0.4 \mathrm{keV}$ is diminished by more than two orders of magnitude. 


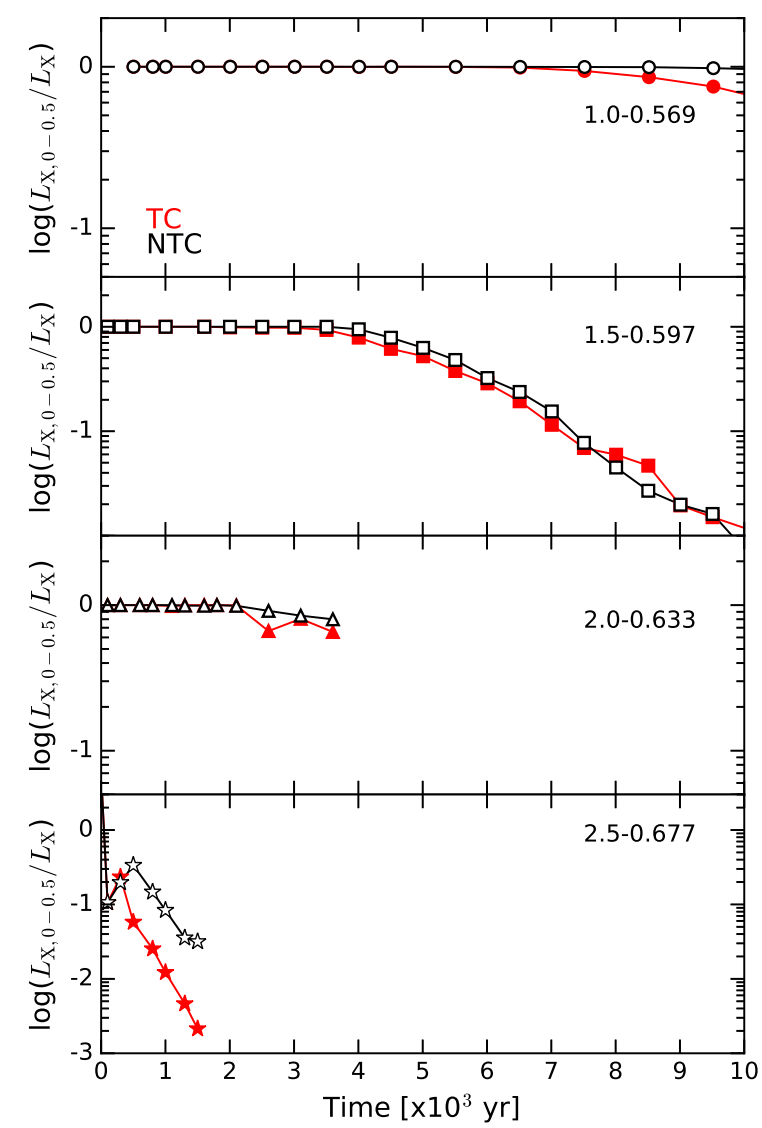

Figure 15. Evolution of the X-ray luminosity with time for gas with scalar values $0<s<0.5$ (shocked fast stellar wind) compared to the total X-ray luminosities in the $0.3-2.0 \mathrm{keV}$ energy range. Filled and open symbols represent models with (TC) and without thermal conduction (NTC), respectively.

Figure 17 shows the result of convolving the absorbed spectrum shown in Figure 16 (moderate absorption) with the response matrices from the Chandra ACIS-S and also XMM-Newton EPIC-pn X-ray camera: 6 , binned with a resolution of $60 \mathrm{eV}$. Note that most of the rich detail of the spectral lines is lost and only a few features can be identified in the spectra. For example, there are indications of the $\mathrm{C}$ VI line at $0.37 \mathrm{keV}, \mathrm{C}_{\mathrm{VI}}$ and the $\mathrm{N}$ vi He-like triplet at $\sim 0.43 \mathrm{keV}$ and the $\mathrm{O}$ VII triplet at $\sim 0.58 \mathrm{keV}$ together with a possible contribution from $\mathrm{O}$ VIII at $\sim 0.65 \mathrm{keV}$. Both the binning and the convolution with the instrumental response matrices combine contributions from various lines in a single bin. Recall that these spectra were generated using the Cloudy standard PN abundance set. If other abundances are used the relative line strengths will be different.

Even though XMM-Newton possesses a higher sensitivity towards lower energies, the following analysis will focus

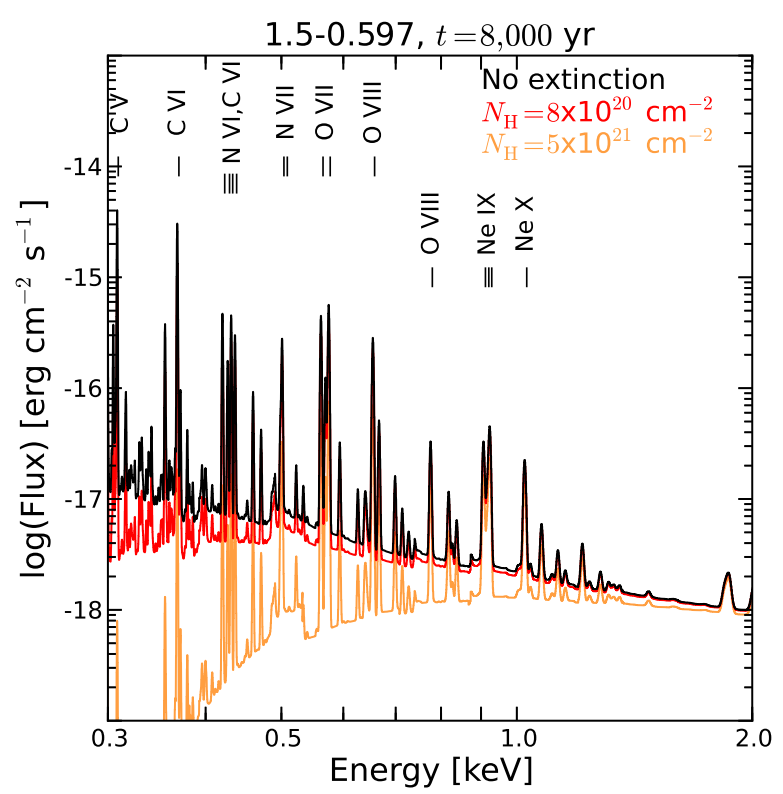

Figure 16. Synthetic spectra generated using the CHIANTI software for the 1.5-0.597 model without thermal conduction at $8000 \mathrm{yr}$ of post-AGB evolution with different interstellar absorbing column densities. The principal emission lines are identified.

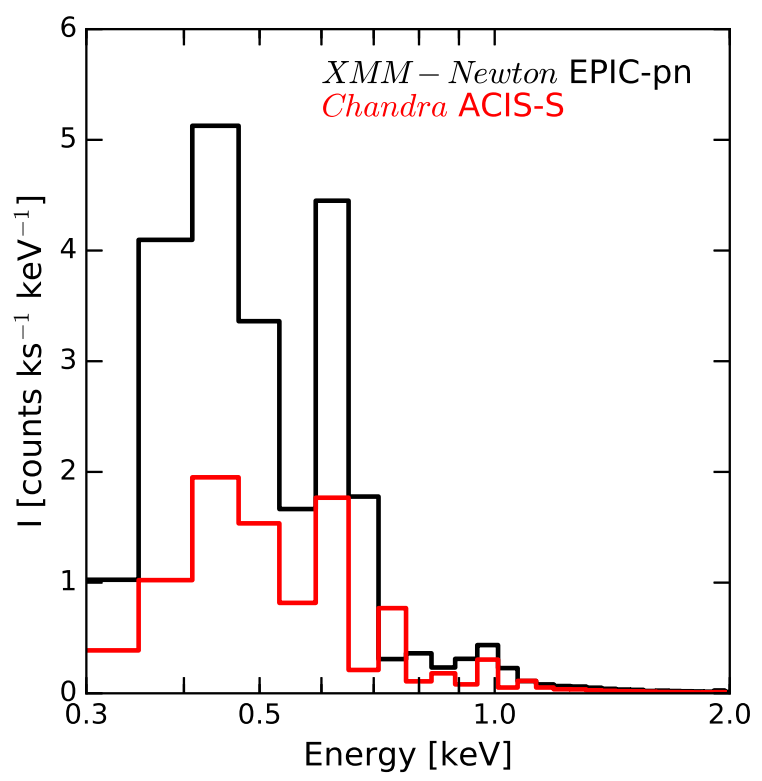

Figure 17. Synthetic spectra for the 1.5-0.597 model at $8000 \mathrm{yr}$ after the onset of the post-AGB wind without thermal conduction (see Fig. 16). The spectra have been convolved with the Chandra (red) and XMM-Newton EPIC-pn camera with the medium optical filter (black) response matrices using spectral bins of width $60 \mathrm{eV}$ and assuming an absorption column density of $N_{\mathrm{H}}=8 \times 10^{20} \mathrm{~cm}^{-2}$. 
on spectra convolved with the Chandra ACIS-S response matrices, in order to facilitate direct comparison with current and future results of the Chandra Planetary Nebula Survey (ChanPlanS; Kastner et al. 2012, Freeman et al. 2014, Montez et al. 2015).

Figure 18 shows examples of the final absorbed and convolved spectra for cases with and without thermal conduction for the 1.5-0.597 models at a selection of different times in the post-AGB evolution. For the earliest time (left-most panels), corresponding to $2000 \mathrm{yr}$ after the onset of the fast wind, results are presented for both moderate $\left(N_{\mathrm{H}}=8 \times 10^{20} \mathrm{~cm}^{-2}\right)$ and high $\left(N_{\mathrm{H}}=5 \times 10^{21} \mathrm{~cm}^{-2}\right)$ neutral hydrogen absorbing column densities. These figures illustrate very clearly the quenching effect that a high interstellar neutral absorbing column density has on these very soft spectra at energies below $0.6 \mathrm{keV}$ : all evidence of the $\mathrm{N}$ vi line has disappeared and it is difficult to distinguish between different models.

It can also be seen in Figure 18 that the count rate generally increases with time, simply reflecting the increase in the DEM with time of the 1.5-0.597 models (see Fig. 5), which also results in the time increase in the total luminosity (see Fig. 11). Moreover, the spectra for the cases with conduction have higher count rates, by up to a factor of 4 , than their counterparts without conduction. This is entirely consistent with the relative DEM values shown in Fig. 5 .

For the models without conduction (Fig. 18 upper panels), the change in the spectral shape from 2000 to 4000 to 8000 yrs reflects the changing DEM of these models (see Fig. 5). At 2000 yrs, the hottest gas has a temperature $\log T \sim 6.3$ and is shocked fast stellar wind material. This gas is the main contributor to the diffuse X-ray emission since it coincides with the peak of the emission coefficient (see Fig. 6). At 4000 yrs the average temperature of the gas reaches its peak (see Fig. [7), although the main contribution to the diffuse X-ray emission still comes from gas with temperature around $\log T \sim 6.3$, at which temperature the turbulent mixing layer gas is the main component (cf. Fig. 14). Higher temperature gas now also contributes to the X-ray emission, and this is reflected in the shape of the spectrum. At the latest time shown (8000 yrs), although the average temperature is very similar to that at 2000 yrs (see Fig. 7), the main contributor to the X-ray emission is the lower temperature gas in the turbulent mixing layer. The main peaks in the absorbed, convolved spectra correspond to emission lines. Higher temperature gas has an increased O VII $(\sim 0.58 \mathrm{keV})$ to $\mathrm{N}$ VI $(\sim 0.43 \mathrm{keV})$ line ratio.

For the 1.5-0.597 models with thermal conduction (Fig. 18, lower panels), the shape of the DEM does not change much from 2000 to 4000 to 8000 yrs, but it does increase in magnitude (see Fig. [5 and 9). This is simply a consequence of the expansion of the hot bubble and the resulting larger emission measure of gas at soft X-ray emitting temperatures.

It is worth mentioning here that, even though we are not tailoring the abundances or morphology to any particular PN, Fig. 18] shows similar spectral features to Chandra observations reported by Chu et al. (2001), Gruendl et al. (2006), and Ruiz et al. (2013) for the PNe NGC 6543, NGC 7026 and NGC 2392, although differences in the binning make a direct comparison difficult. In this paper, a single set of abundances has been used for both fast stellar wind and nebular components but it should be appreciated that if different abundance sets are used for the two components, then this would also have an effect on the evolution of the spectral shape.

\subsection{Sampled spectra}

It should be borne in mind that the absorbed, convolved, synthetic spectra, such as those presented in Figure 18 (solid lines), correspond to ideal spectra, i.e., as if they were obtained by infinite-exposure time observations. A real observation lasts a finite amount of time. It is possible to mimic finite-time observations by sampling the synthetic spectrum to imitate photons (counts) in a real observation.

In order to simulate an observation, the cumulative absorbed spectrum is randomly sampled in the $0.3-2.0 \mathrm{keV}$ energy range, according to the desired photon count number. The process was repeated $10^{4}$ times in order to generate independent selections of a given photon count number. The standard deviations for 50 and 200 photon count samples are shown in Fig. 18 by grey and black error bars, respectively, where we consider only the case of a moderate absorbing column density $\left(N_{\mathrm{H}}=8 \times 10^{20} \mathrm{~cm}^{-2}\right)$.

Figure 18 shows that observations with 50 counts are not enough to give a fair description of the true spectral shape when the binning is $60 \mathrm{eV}$. A coarse binning would be recomended for such a low number of total counts. Hence the physical properties of hot bubbles in PNe derived from such low count-rate spectra will not be very reliable. Unfortunately, there are several PNe that have not been observed with more than 50 counts due to their reduced exposure times (see Kastner et al. 2012; Ruiz et al. 2013; Freeman et al. 2014). On the other hand, 200-count spectra give a reasonable description of the spectral shape. Hence the physical properties derived from these spectra will be more representative of the real conditions in the hot bubble gas.

This is a very interesting subject. In addition, different abundances in the nebular and fast stellar wind gas could be taken into account in the spectral calculation and the absorbing column density, $N_{\mathrm{H}}$, could be varied. Such a detailed analysis will be pursued in a subsequent paper.

\subsection{X-ray surface brightness}

As an additional tool in the comparison with observations, we can create surface brightness profiles of the soft X-ray emission from the hot bubbles. For this, we have integrated the X-ray intensity in the $0.3-2.0 \mathrm{keV}$ energy band, taking into account the absorption by the neutral interstellar medium. Furthermore, all profiles must be convolved with the spatial response of the telescope, which is a combination of the instrumental PSF and smoothing kernel. This can be simulated by using a Gaussian profile.

Figure 19 (top panel) presents the resulting normalized surface brightness profiles from the 1.5-0.597 models with and without thermal conduction at the time when the hot bubble has a mean radius of 0.2 pc (see Figs. 1 and 2). An absorbing neutral hydrogen column density of $N_{\mathrm{H}}=8 \times 10^{20} \mathrm{~cm}^{-2}$ was assumed. The normalized surface brightness profiles would be described as limb-brightened 

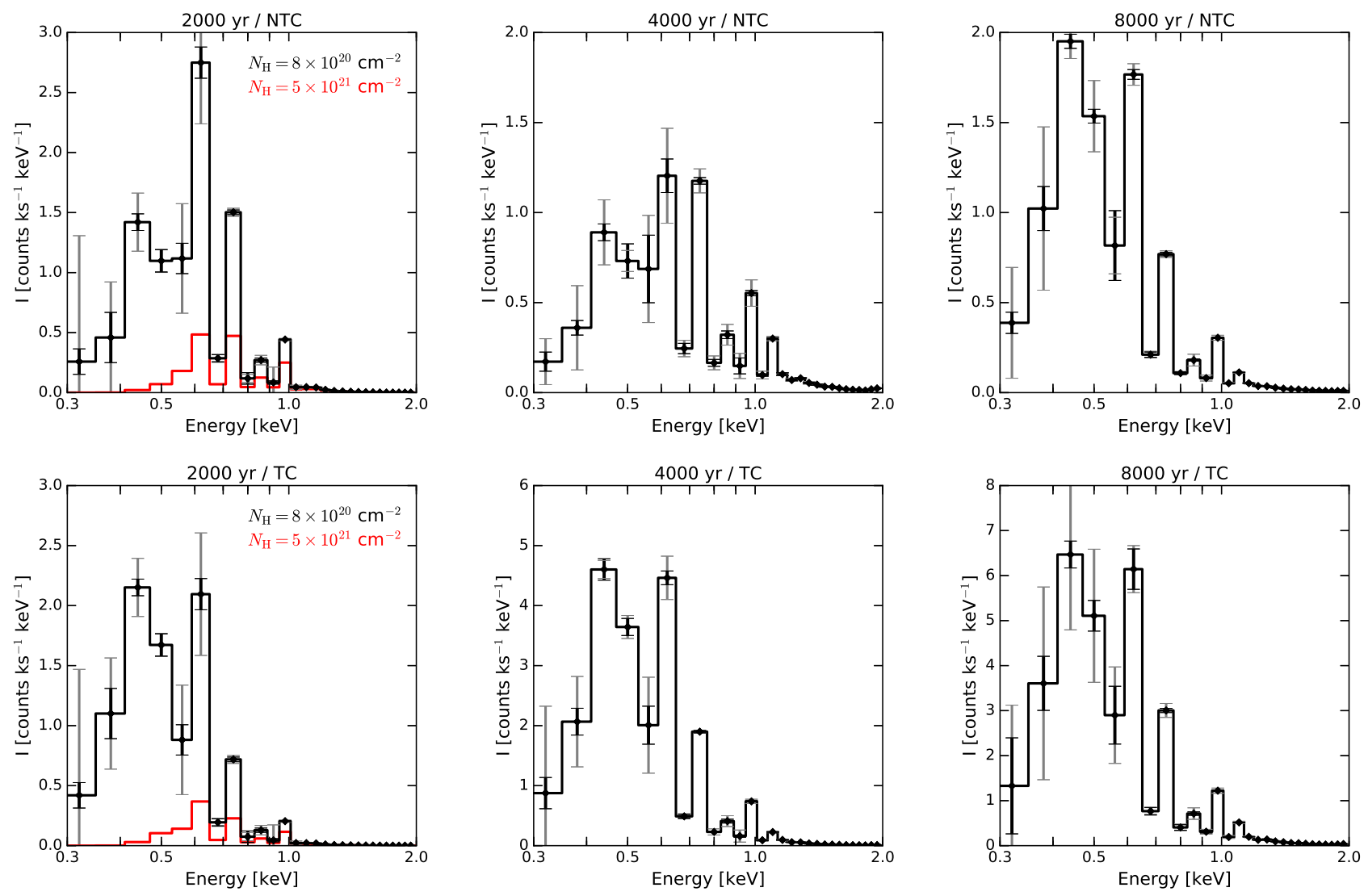

Figure 18. Synthetic X-ray spectra for the 1.5-0.597 models with (TC) and without thermal conduction (NTC) at 2000, 4000 and $8000 \mathrm{yr}$ after the onset of the post-AGB phase. Black and red lines represent two values of the absorbing neutral hydrogen column densities: moderate $8 \times 10^{20} \mathrm{~cm}^{-2}$, and high $5 \times 10^{21} \mathrm{~cm}^{-2}$, respectively. Grey and black error bars correspond to spectral sampling of 50 and 200 counts, respectively (see text for details). All spectra were convolved with the Chandra ACIS-S response matrices using a $60 \mathrm{eV}$ bin width.

for the graphs shown in the top panel. However, the radial positions of the peaks of the profiles are not at the edge of the bubbles, as might be expected, but at $65-75 \%$ of the radius. This is because the soft $\mathrm{X}$-ray emission produced by cooler gas, which is found around the edge of the hot bubble, is more affected by absorption than the X-ray emission from hotter gas (see, e.g., Figure 16) as described by Steffen et al. (2008). We note that the unsmoothed surface brightness profiles from both models present structure in their inner regions. This is due to the large-scale non-radial motions in these simulations, which drag material from the shell into the inner part of the hot bubble, as seen in middle panels of Figures 1 and 2 .

The other panels in Figure 19 show the results obtained from applying Gaussians with different FWHM to the same profiles shown in the top panel. Each value of the FWHM represents a fraction of the effective radius of the hot bubble. The effect of applying a heavy smoothing process (higher FWHM) can give the appearance that the X-ray-emitting gas is distributed with a maximum at the center of the hot bubble, i.e., has a flatter surface brightness distribution. This smoothing process may be unavoidable in low-countnumber detections of PNe.

\section{DISCUSSION}

Our work in this paper confirms that thermal conduction can be an important physical process for the production of soft, diffuse X-ray emission in PNe. However, instabilities, which form in the swept-up shell as the fast stellar wind accelerates into the expelled envelope (i.e., nebular) material, are also of great importance especially if conduction is suppressed or absent. The clumps and filaments in the broken shell can act as sources for photoevaporated flows and hydrodynamic mixing layers. The gas in the turbulent mixing layers can be shock-heated to averaged temperatures of $T_{\mathrm{A}} \sim 10^{6} \mathrm{~K}$. We find that the characteristic temperature of the X-ray emitting gas reported by observations is well approximated by the mean emissivity weighted temperature of the hot bubble ( $T_{\mathrm{A}} \approx T_{\mathrm{X}}$; see Fig. 8$)$. The combination of the emissivity coefficient, which for planetary nebula abundances peaks about $\log T \sim 6.3$ and the differential emission measure distributions, which are highest for $\log T \leqslant 6$, results in a mean temperature precisely within the observed range. The hydrodynamic mixing layer results give higher temperatures than the thermal conduction results.

For the four initial stellar masses considered in this paper, the brightest X-ray objects are the shortest lived, for example the X-ray luminosity of the 2.5-0.677 model fades within $2000 \mathrm{yrs}$, while the luminosity of the 1.0-0.569 


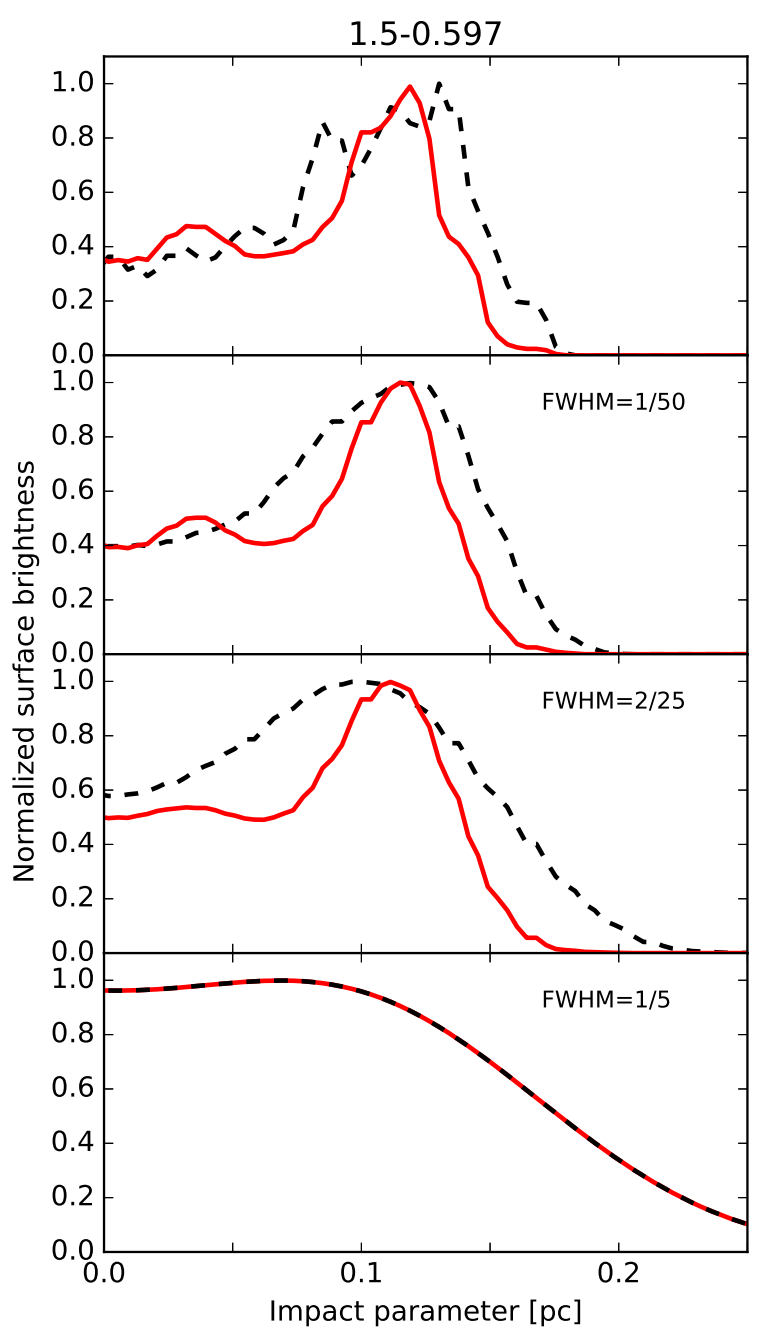

Figure 19. Normalized surface brightness profiles in the 0.3$2.0 \mathrm{keV}$ energy range for the 1.5-0.597 model for the time when the rim shell is $\sim 0.2 \mathrm{pc}$ with interstellar absorption of $N_{\mathrm{H}}=$ $8 \times 10^{20} \mathrm{~cm}^{-2}$ for different Gaussian convolutions. Dashed (black) lines represent the results without thermal conduction and solid (red) lines the results with thermal conduction. Each value of the FWHM represents a fraction of the effective radius of the hot bubble.

model, although low, continues at the same level for more than 10,000 yrs. However, the surface brightness of the longer-lived models decreases with time as the size of the hot bubble increases, and this could influence the detection of these objects. Our numerical results are also consistent with the sizes of $\mathrm{PNe}$ detected in diffuse X-rays: the bright, short-lived X-ray emission comes from hot bubbles smaller than $R_{\mathrm{HB}} \sim 0.1 \mathrm{pc}$ and corresponds to the most massive stars, while larger $\left(R_{\mathrm{HB}}>0.1 \mathrm{pc}\right)$, less bright hot bubbles are produced by the more slowly evolving lowermass objects. Our models would seem to predict far more large, low brightness objects than are actually observed. The lower limits for the X-ray luminosity and surface brightness of these models correspond to the cases without conduction and are an order of magnitude smaller. It is possible that we are observationally limited by the current generation of $\mathrm{X}$-ray satellites. Detections of surface brightness levels below $10^{-17} \mathrm{erg} \mathrm{s}^{-1} \mathrm{~cm}^{-2} \operatorname{arcsec}^{-2}$ will be achieved with the Athena+ telescope (Nandra et al. 2013).

We find that at early times all of our models predict that the X-ray emission will be dominated by the shocked fast wind material but at intermediate and later times the nebular contribution dominates. This is consistent with the previous theoretical work of Akashi et al. (2007), who showed that diffuse X-ray emission can come from a shocked stellar wind whose velocity is rapidly increasing in the first $1000 \mathrm{yrs}$ of post-AGB evolution.

Although we assume in our models a single set of abundances for both the nebular and CSPN fast wind material, in reality these could be different, with the CSPN abundances being metal-enriched due to the expulsion of the hydrogenrich envelope (Georgiev et al. 2008; Morisset \& Georgiev 2009). Thus, the change in the relative importance of the hot, shocked stellar wind gas and the heated nebular material seen in Figure 15 should also manifest itself as an evolution of the abundances responsible for the X-ray spectra of these objects. For instance, at early times we could expect the abundances to be dominated by hot, shocked stellar wind material, while at later times nebular abundances will determine the spectral characteristics. This is more relevant for the case of PNe with [WR]-type nuclei and has been recently explored by Sandin et al. (2016).

There is some observational evidence that very young $\mathrm{PNe}$ do indeed show X-ray spectra that can be well fitted as a hot plasma with stellar abundances, suggesting that the stellar wind dominates the X-ray emission in these objects. Even though it is a short-lived phase, and even shorter in the most massive cases, there is one observed object, $\mathrm{BD}+30^{\circ} 3639$, that requires stellar abundances, since $\mathrm{C}$ and $\mathrm{Ne}$ are enhanced. Yu et al. (2009) analyzed Chandra Low Energy Transmission Gratings (LETG) spectra in conjunction with the ACIS-S data. The high-quality LETG/ACIS$\mathrm{S}$ spectra enabled the authors to conclude unambiguously that the diffuse X-ray emission in this PN has abundances close to those of the CSPN. The second brightest X-ray PN, NGC 6543, on the other hand, does not show any evidence for enhanced $\mathrm{C}$ and $\mathrm{Ne}$, and so nebular abundances are more appropriate for this object (Guerrero et al. 2015), although higher signal-to-noise observations are required to confirm this statement. Both observations are fully consistent with our numerical models, particularly since the hot bubble in NGC 6543 is larger than that of $\mathrm{BD}+30^{\circ} 3639$ and it is assumed to be an older object.

In the presentation of our results, we have taken care to include observational effects so that our models can be compared directly with, for example, the results from the CHANPlaNS survey. In particular, we show that the radial surface brightness profile that is obtained from a given hot bubble depends very much on the size of the hot bubble combined with the instrumental PSF with which the models are convolved. The same model can give a limb-brightened or flat surface brightness profile depending on the FWHM of the Gaussian profile of the PSF. Indeed, observations of the same object with XMM-Newton and Chandra can give different radial surface brightness profiles, such as is the case 
with NGC 3242 (see Ruiz et al. 2011. Kastner et al. 2012). This is because the PSF of the EPIC XMM-Newton X-ray detectors is not able to resolve structures with the same level of detail as the back-illuminated ACIS-S CCD3 of the Chandra telescope. Given that the Chandra observations of diffuse X-rays in PNe are only marginally resolved in the majority of cases (small angular size) and in many cases have a low count rate, it is unwise to attribute a particular morphology to the X-ray emitting gas.

In a similar spirit, we have simulated finite-time observations by sampling the ideal spectrum a fixed number of times. From these experiments, we conclude that observed spectra with a low total number of counts do not reliably describe the spectral shape and hence should not be used to derive the physical parameters (temperature, abundances, absorbing column density of neutral gas) of the observed hot bubble. We suggest that at least 200 total counts are needed per object.

At the earliest times, the hot bubble in our models does not occupy a large fraction of the computational grid. For this reason, the results for the smallest bubbles are not as detailed as at later times, when the bubbles are larger and the structures are resolved with more computational cells. As part of our ongoing investigations into the production of diffuse X-ray emission in planetary nebulae, we will study the earliest stages of the hot bubble evolution at higher numerical resolution in future work. This is particularly important since the brightest and best studied observed X-ray PN have small radii $\left(R_{\mathrm{HB}}<0.1 \mathrm{pc}\right)$. Increasing the numerical and temporal resolution at the earliest stages of high initial stellar mass models $\left(2.0 M_{\odot}<M<2.5 M_{\odot}\right)$ will also permit us to characterise the fragmentation of the swept-up shell due to instabilities in the wind-wind interaction region. It would also be interesting to include a larger number of stellar models in this mass range.

Finally, although our results with thermal conduction are consistent with a full range of observed parameters and trends, it is worth mentioning that even when magnetic fields are present in a PN and conduction can be assumed to be suppressed, soft diffuse X-ray emission from the hot bubbles can be expected in the majority of cases due to the turbulent mixing layers. Stute \& Sahai (2006) have pointed out that even small magnetic fields of the order of $\mu \mathrm{G}$ would be able to suppress conduction by thermal electrons in the direction perpendicular to the magnetic field lines. Recent, low-resolution, spectropolarimetric observations of a sample of CSPN in a variety of different PN morphologies do not find significant evidence for surface magnetic fields in the central stars Steffen et al. 2014). Any magnetic field in the PN itself must therefore be already present in the expelled envelope. Polarization of thermal molecular lines of $\mathrm{CO}$ and $\mathrm{SiO}$ in the envelopes of AGB stars (Vlemmings et al. 2012) and also maser studies of these objects (Vlemmings 2007) show that magnetic fields are generally present at this stage of evolution. Dust polarization studies of two protoplanetary nebulae show wellorganized magnetic fields aligned with molecular outflows in these objects (Sabin et al. 2014). Indeed, magnetic fields are one of the possible mechanisms for shaping bipolar nebulae. Polarized dust also provides evidence for magnetic fields in the bipolar PNe NGC6302, NGC 6537, and NGC 7027 (Sabin. Ziilstra. \& Greaves 2007), however, this sort of plan- etary nebula is least likely to show diffuse X-ray emission (Kastner et al. 2012; Freeman et al. 2014). Thus, fossil magnetic fields could be present in the nebular gas surrounding the CSPN at the onset of the fast wind. The high incidence of diffuse X-ray detections in $\mathrm{PNe}(\sim 30 \%$; Kastner et al. 2012; Freeman et al. 2014) therefore requires explanations that do not necessarily require thermal conduction.

\section{SUMMARY AND CONCLUSIONS}

In this paper, we have presented calculations of the synthetic diffuse X-ray emission from hot bubbles in PNe from the results of $2 \mathrm{D}$ axisymmetric high-resolution, radiationhydrodynamic simulations presented in Toalá \& Arthur (2014). The numerical results correspond to models with initial masses (M $\mathrm{M}_{\mathrm{ZAMS}}$ ) of $1,1.5,2$, and $2.5 \mathrm{M}_{\odot}$, which have final WD masses of $0.569,0.597,0.633$, and $0.677 \mathrm{M}_{\odot}$, respectively. The density and temperature information from the numerical simulations are used to compute the differential emission measure (DEM) distribution of the gas and study its evolution with time. The DEM distributions were used to compute synthetic spectra in the $0.3-2.0 \mathrm{keV}$ energy range making use of CHIANTI software. This allows us to study the time evolution of the spectra of each model. Luminosities were obtained from integrating the spectra. In addition, the use of an advected scalar in the numerical simulations enables us to separate out the contribution of the shocked fast stellar wind material from that of the swept-up nebular gas to the X-ray emission.

Our main findings can be summarized as follows:

(i) Instabilities created in the wind-wind interaction zone at the contact discontinuity between the fast stellar wind and the nebular gas lead to the formation of clumps and filaments whose large surface area results in extensive turbulent mixing layers or regions of thermally evaporated nebular material having densities and temperatures intermediate between the tenuous hot, shocked fast wind plasma and the dense photoionized nebular material. This is the gas responsible for producing the soft X-ray emission, whose spectra and luminosities agree nicely with observations. Cases in which thermal conduction is included have higher fluxes and luminosities than cases with purely hydrodynamic mixing layers.

(ii) The differential emission measure distributions (DEMs) from models without thermal conduction are different to those from models with conduction. When there is no conduction, the DEM distribution falls monotonically from high DEM values at low plasma temperatures to low DEM values a high plasma temperatures. In models with thermal conduction, heat diffusion transfers heat from the hottest gas to the gas at the interface between the hot bubble and the sweptup nebular shell and, increases the DEM values for all intermediate temperatures. A plateau of roughly constant DEM value forms at temperatures $T<10^{6} \mathrm{~K}$, which corresponds to nebular gas in the mixing layer.

(iii) The emissivity weighted mean plasma temperatures of all our models lie within derived values of the temperature of observed X-ray-emitting gas in PNe. This 
is a consequence of a sharply peaked emission coefficient and DEM distributions that increase towards lower temperatures. Models with thermal conduction show very uniform values of the mean temperature, both as a function of time and between models for different stellar masses. This suggests that the properties of the conduction layer are nearly independent of the stellar parameters. The mean temperatures of the models without conduction show more variation and reach higher values at early times. They fall off to temperature levels obtained for models with thermal conduction once the mixing layer begins to dominate.

(iv) At early times, the diffuse X-ray emission is provided by shocked fast stellar wind material. For the models with thermal conduction, the contribution of the evaporated nebular material to the diffuse X-ray emission becomes dominant ( $>50 \%$ ) well before peak luminosity is reached. For the models without thermal conduction, nebular material heated in the turbulent mixing layers can dominate the diffuse X-ray emission around peak luminosity. This suggests that stellar abundances should be appropriate for the diffuse X-ray emission from very young, compact $\mathrm{PNe}$, but that X-ray emission from older PNe would be better fit with nebular abundances.

(v) Our results suggest that the higher initial mass stellar models $M>2.0 M_{\odot}$ produce a better fit to the current detected objects since they produce small, X-ray luminous bubbles. It would be worthwhile to investigate these models at higher spatial and temporal resolution and with a fuller coverage of the initial stellar mass parameter space.

(vi) Using sampled spectra to represent finite time observations, we have shown that 200 counts or more are necessary to give a reliable description of the spectral shape, from which the physical properties of the hot plasma in PNe can be derived. The spectra from our models with thermal conduction are all very similar, but spectra from models without conduction are much more varied.

(vii) The radial surface brightness profile of an observed planetary nebula has as much to do with the instrumental PSF and whether the angular size of the object is resolved, as with the actual distribution of the X-ray emitting gas. For this reason, it is unreasonable to discard models that do not produce flat X-ray surface brightness profiles. Once a limb-brightened theoretical radial surface brightness profile is smoothed to the same resolution as the observations, it too can appear to be flat.

\section{ACKNOWLEDGMENTS}

We are thankful to our referee, Dr. Detlef Schönberner, who performed an outstanding job that helped improve our results and the clarity of this paper. We would like to thank M. A. Guerrero and W. J. Henney for fruitful discussions during the realization of this work. SJA and JAT acknowledge financial support through PAPIIT project IN101713 from DGAPA-UNAM (Mexico). JAT thanks support by CSIC JAE-Pre student grant 2011-00189 (Spain). JAT was

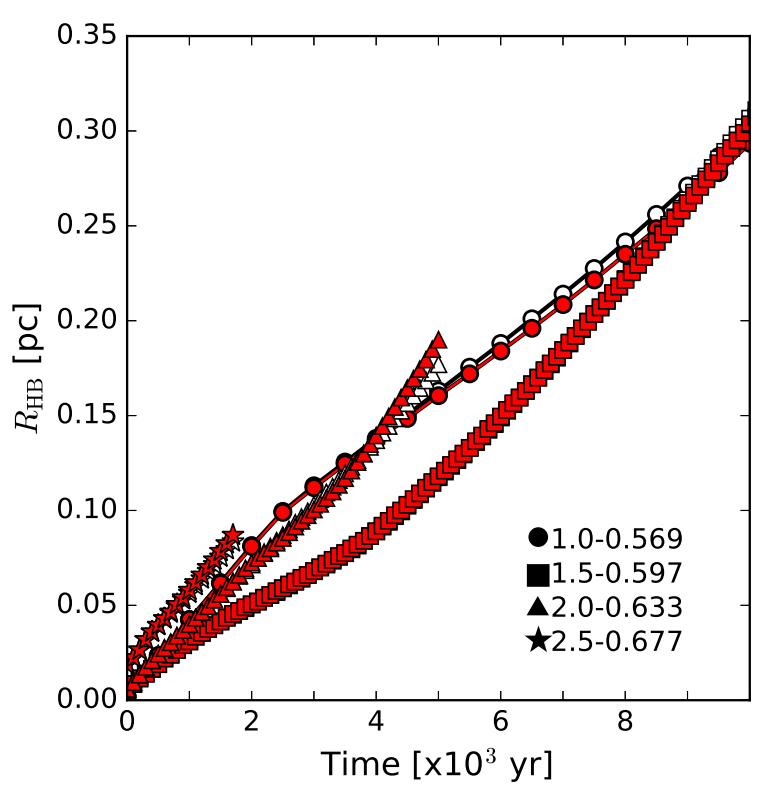

Figure B1. Mean radius of the hot bubble as a function of time for the three principal stellar models for the 1D simulations. Filled symbols are for the models that include thermal condutcion, while open symbols are for the models that do not.

also supported by AYA 2011-29754-C03-02 of the Spanish Ministerio de Economía y Competitividad (MINECO) cofunded by FEDER funds.

\section{APPENDIX A: STELLAR PARAMETERS}

In this appendix we present the stellar wind parameters, that is, terminal wind velocity $\left(v_{\infty}\right)$ and mass-loss rate $(\dot{M})$, and also the ionizing photon flux $\left(Q_{\mathrm{H}}\right)$ for the different models used in the present paper. Note that very similar figures have been presented in Paper I. As mentioned in Paper I, all of our stellar wind parameters and ionization photon fluxes were computed using the stellar atmosphere code WM-BAsIC (see Pauldrach et al. 2012, and references therein). Figure A1 also presents the evolution of the mechanical luminosity as a function of the effective temperature of each model.

In all our simulations the zero point of evolution $t=0$ corresponds to $\log \left(T_{\text {eff }}\right)=4$ at the onset of the post-AGB phase, as defined by the stellar evolutionary models of Vassiliadis \& Wood (1994) (see Paper I).

\section{APPENDIX B: ONE DIMENSIONAL RESULTS}

In this appendix we present the results for models with and without thermal conduction as obtained from the $1 \mathrm{D}$ version of our radiation-hydrodynamic code. These simulations were performed at the same numerical resolution as the $2 \mathrm{D}$ models.

First, we show the expansion of the hot bubble as a function of time. For the 1D models this is simply the position of the contact discontinuity as a function of time and is shown in Figure B1 The variety of expansion behaviours is due to 

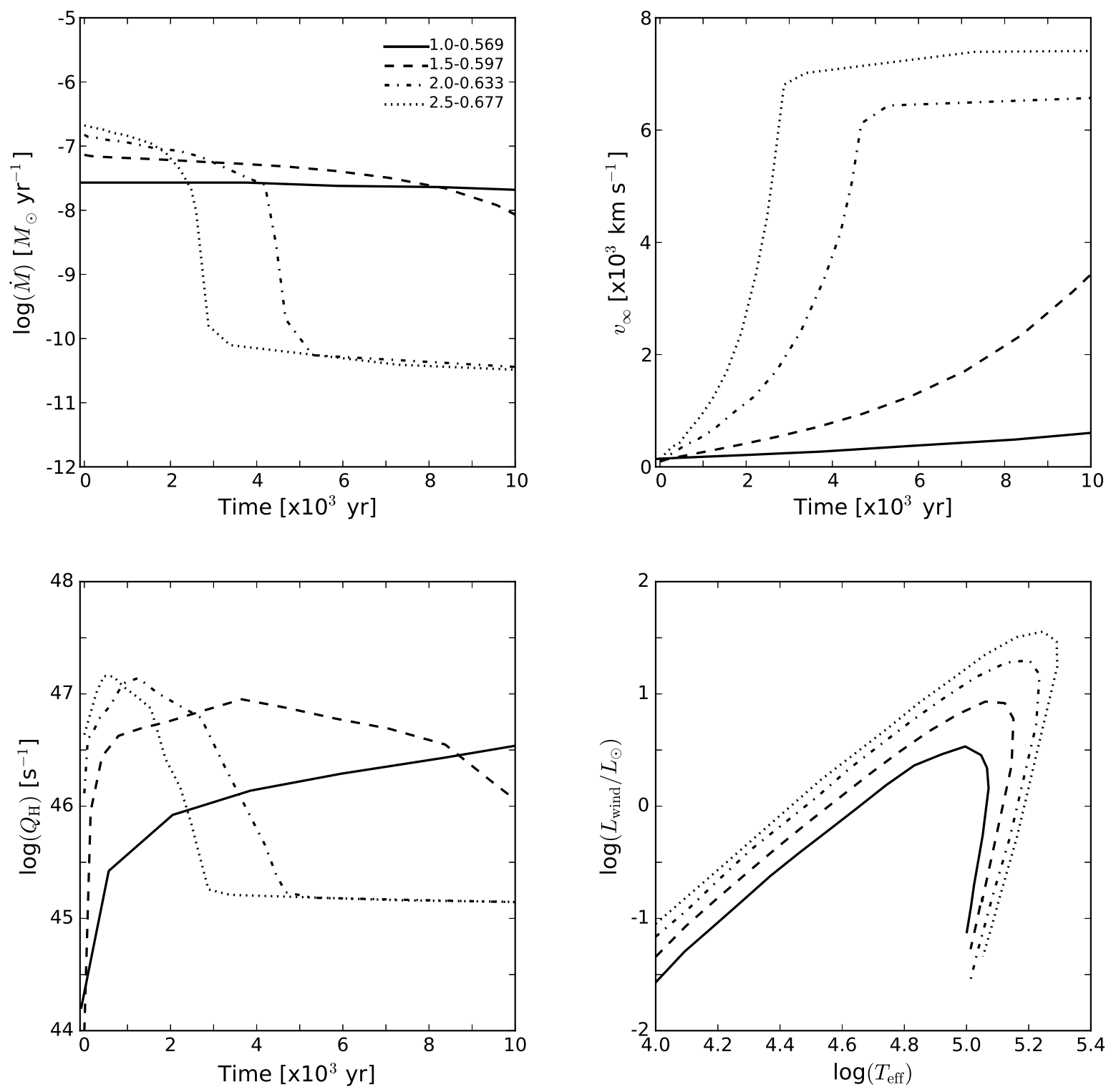

Figure A1. (Upper left) Mass-loss rate, (upper right) stellar wind velocity, and (bottom left) ionizing photon flux as a function of time, and (bottom right) stellar wind luminosity as a function of effective temperature for the different stellar models used in this work. The initial-final mass of each model is marked on the upper left panel.

the different stellar wind histories and initial density distributions of the different models. As shown by Steffen et al. (2008) the cases with and without thermal conduction evolve virtually at the same rate.

We focus particularly on the 1.5-0.597 models, as was done for the 2D models in the main paper. Figure B2 top panel presents examples of the DEM at $[2,4$, and 8$] \times 10^{3} \mathrm{yr}$. This figure shows that for the case without thermal conduction the DEM is restricted to discontinuous, narrow temperature ranges: the lower temperature gas comes from a few dense cells at the contact discontinuity whose relative contribution would be lower if a higher grid resolution were used. The higher temperature DEM comes from the shocked fast stellar wind that fills most of the volume of the hot bubble.
Only the latest time results show the DEM distributed over a wider temperature range.

On the other hand, the 1D cases with thermal conduction (see Fig. B2) show a more continuous distribution similar to those presented in Fig. 5 (although for gas with $\log T<6$ the temperature bin resolution has an effect). This is because gas at this temperature belongs to the conduction layer; in the $2 \mathrm{D}$ case the instabilities increase the surface area of the contact discontinuity, and hence the quantity of gas in the conduction layer as compared to the 1D case.

Figure B3 shows the average temperature of both sets of $1 \mathrm{D}$ simulations calculated using Eqs. 1 and 2 The average temperature of the 1D simulations without conduction starts at $\log T_{\mathrm{A}}=6$ but ends up much higher, with $\log T_{\mathrm{A}} \sim 7$, far higher than any temperature reported from observations. 


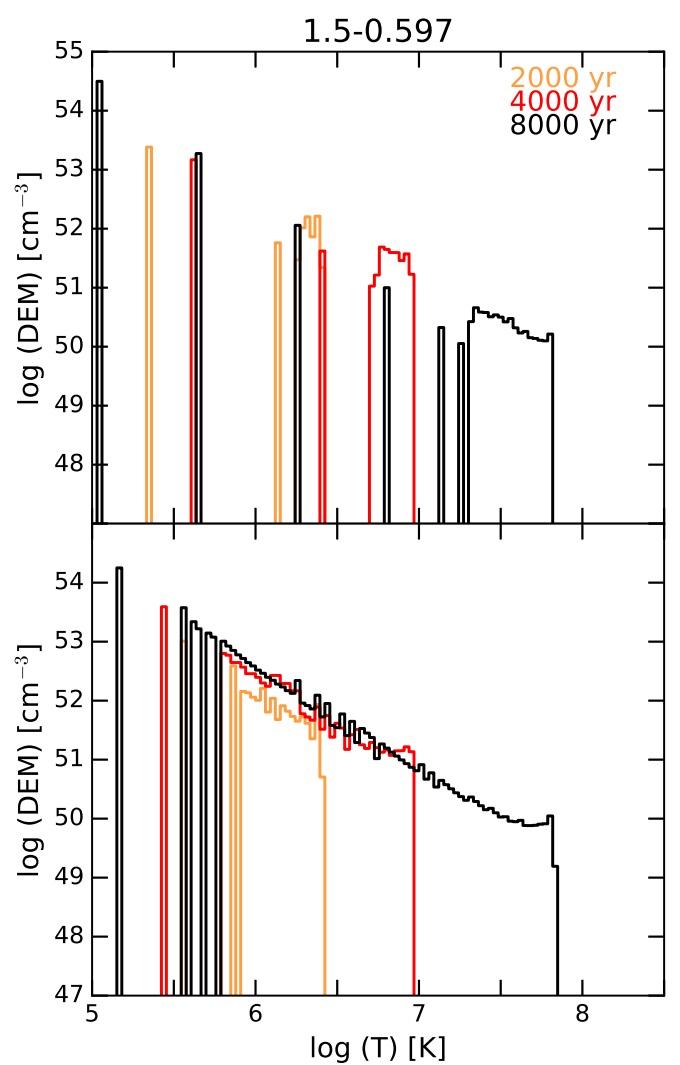

Figure B2. Differential emission measure (DEM) for the 1.50.597 model as computed from 1D numerical results without (top panels) and with (bottom panels) thermal conduction. The colours represent different times, as indicated in the upper panel.

The average temperature of the $1 \mathrm{D}$ simulations with conduction hovers around $\log T_{\mathrm{A}}=6.3$. Even in this case the average temperature is higher than the corresponding $2 \mathrm{D}$ results.

Fig. B4 shows the corresponding spectra, computed using CHIANTI, resulting from the DEMs of Fig. B2, The shape of the spectra from the $1 \mathrm{D}$ simulations without conduction is very flat in the $0.3-2.0 \mathrm{keV}$ energy range due to the much higher gas temperatures. The fluxes at low energy for the 1D cases with conduction are an order of magnitude higher than those without conduction. The fluxes of the 1D simulations are more varied than the $2 \mathrm{D}$ cases because there is not the continuum in the DEM distribution that we obtain in the $2 \mathrm{D}$ models.

\section{REFERENCES}

Akashi, M., Meiron, Y., \& Soker, N. 2008, New Astron., 13,563

Akashi, M., Soker, N., Behar, E., \& Blondin, J. 2007, MNRAS, 375, 137

Balick, B. 1987, AJ, 94, 671

Balucińska-Church, M., \& McCammon, D. 1992, ApJ, 400, 699

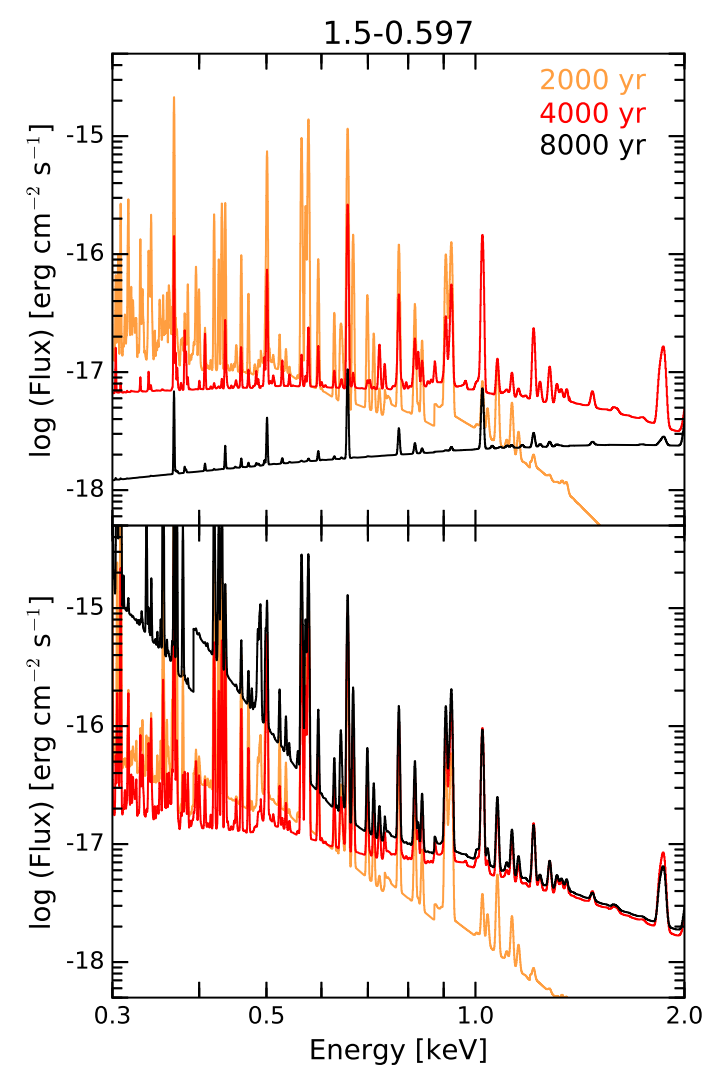

Figure B4. Spectra generated from the DEMs presented in Fig. B2 for the 1D numerical results without (top panels) and with (bottom panels) thermal conduction. The colours represent the same times as indicated in Fig. B2

Chu, Y.-H., Guerrero, M. A., Gruendl, R. A., Williams, R. M., \& Kaler, J. B. 2001, ApJL, 553, L69

Dere K. P., Landi E., Mason H. E., Monsignori Fossi B. C., Young P. R., 1997, A\&AS, 125, 149

Dere, K. P., Landi, E., Young, P. R., et al. 2009, A\&A, 498, 915

Dyson, J. E., \& Williams, D. A. 1997, The physics of the interstellar medium. Edition: 2nd ed. Publisher: Bristol: Institute of Physics Publishing, 1997. Edited by J. E. Dyson and D. A. Williams. Series: The graduate series in astronomy. ISBN: 0750303069,

Ferland, G. J., Porter, R. L., van Hoof, P. A. M., et al. 2013, RevMexAA, 49, 137

Freeman, M., Montez, R., Jr., Kastner, J. H., et al. 2014, ApJ, 794, 99

Frew, D. J. 2008, Ph.D. Thesis, Macquarie University, Sidney, Australia

Georgiev L. N., Peimbert M., Hillier D. J., Richer M. G., Arrieta A., Peimbert A., 2008, ApJ, 681, 333

Gruendl, R. A., Guerrero, M. A., Chu, Y.-H., \& Williams, R. M. 2006, ApJ, 653, 339

Guerrero, M. A., Toalá, J. A., Chu, Y.-H., \& Gruendl, R. A. 2015, A\&A, 574, AA1

Guerrero, M. A., \& De Marco, O. 2013, A\&A, 553, A126

Guerrero, M. A., Gruendl, R. A., \& Chu, Y.-H. 2002, A\&A, 


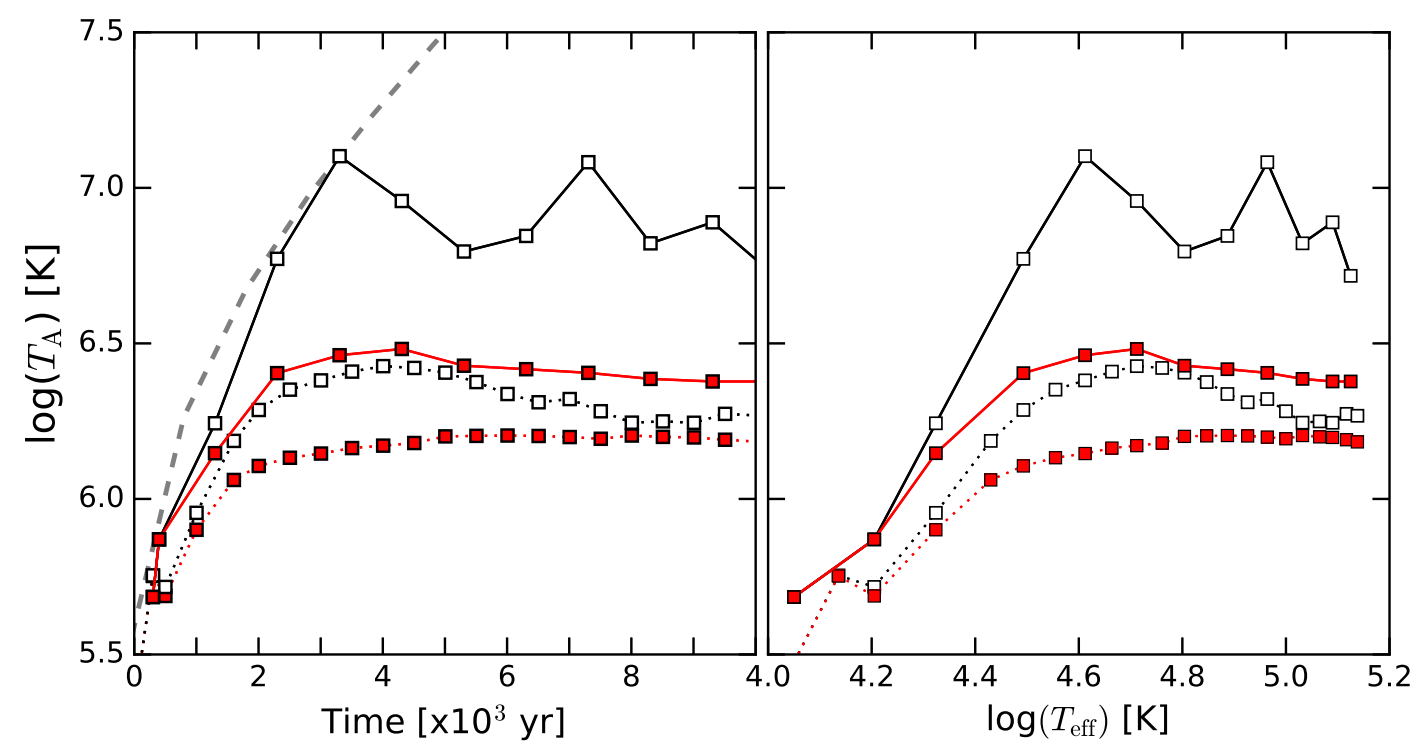

Figure B3. Average temperature for the 1.5-0.597 model from 1D numerical simulations. Left panel: average temperature as a function of time. Right panel: average temperature as a function of stellar effective temperature. The continuous lines with filled symbols are for the case with thermal conduction, the continuous lines with open symbols are for the case without thermal conduction. The dotted lines show the corresponding $2 \mathrm{D}$ results for comparison. The dashed gray line shows the evolution of the post-shock temperature computed as $T=3 \mu m_{\mathrm{H}} v_{\infty}^{2} / 16 k_{\mathrm{B}}$.

387, L1

Herald, J. E., \& Bianchi, L. 2011, MNRAS, 417, 2440

Kastner, J. H., Montez, R., Jr., Balick, B., et al. 2012, AJ, 144,58

Kastner, J. H., Soker, N., Vrtilek, S. D., \& Dgani, R. 2000, ApJL, 545, L57

Kwok, S., Purton, C. R., \& Fitzgerald, P. M. 1978, ApJ, 219, L125

Landi, E., Young, P. R., Dere, K. P., Del Zanna, G., \& Mason, H. E. 2013, ApJ, 763, 86

Li, J., Harrington, J. P., \& Borkowski, K. J. 2002, AJ, 123, 2676

Lou, Y.-Q., \& Zhai, X. 2010, MNRAS, 408, 436

Mellema, G., \& Frank, A. 1995, MNRAS, 273, 401

Marcolino, W. L. F., Hillier, D. J., de Araujo, F. X., \&

Pereira, C. B. 2007, ApJ, 654, 1068

Montez, R., Jr., Kastner, J. H., Balick, B., et al. 2015, ApJ, 800,8

Morisset C., Georgiev L., 2009, A\&A, 507, 1517

Nandra, K., Barret, D., Barcons, X., et al. 2013, arXiv:1306.2307

Pauldrach, A. W. A., Vanbeveren, D., \& Hoffmann, T. L. 2012, A\&A, 538, A75

Pauldrach, A. W. A., Hoffmann, T. L., \& Méndez, R. H. 2004, A\&A, 419, 1111

Pauldrach, A. W. A., Hoffmann, T. L., \& Lennon, M. 2001, A\&A, 375, 161

Pauldrach, A. W. A., Kudritzki, R. P., Puls, J., Butler, K., \& Hunsinger, J. 1994, A\&A, 283, 525

Pauldrach, A. 1987, A\&A, 183, 295

Pauldrach, A., Puls, J., \& Kudritzki, R. P. 1986, A\&A, 164, 86

Perinotto, M., Schönberner, D., Steffen, M., \& Calonaci, C. 2004, A\&A, 414, 993
Ruiz, N., Chu, Y.-H., Gruendl, R. A., et al. 2013, ApJ, 767, 35

Ruiz, N., Guerrero, M. A., Chu, Y.-H., \& Gruendl, R. A. 2011, AJ, 142, 91

Sabin L., Zhang Q., Zijlstra A. A., Patel N. A., Vázquez R., Zauderer B. A., Contreras M. E., Guillén P. F., 2014, MNRAS, 438, 1794

Sabin L., Zijlstra A. A., Greaves J. S., 2007, MNRAS, 376, 378

Sandin, C., Steffen, M., Schönberner, D., Rühling, U. 2016, A\&A, 586, A57

Soker, N. 1994, AJ, 107, 276

Spitzer, L. 1962, Physics of Fully Ionized Gases, New York: Interscience (2nd edition)

Steffen, M., Hubrig, S., Todt, H., et al. 2014, A\&A, 570, AA88

Steffen, M., Schönberner, D., \& Warmuth, A. 2008, A\&A, 489, 173

Stute, M., \& Sahai, R. 2006, ApJ, 651, 882

Toalá, J. A., \& Arthur, S. J. 2014

Vassiliadis, E., \& Wood, P. R. 1993, ApJ, 413, 641

Vassiliadis, E., \& Wood, P. R. 1994, ApJSS, 92, 125

Villaver, E., Manchado, A., \& García-Segura, G. 2002, ApJ, 581,1204

Vlemmings W. H. T., Ramstedt S., Rao R., Maercker M., 2012, A\&A, 540, LL3

Vlemmings W. H. T., 2007, IAUS, 242, 37

Yu, Y. S., Nordon, R., Kastner, J. H., et al. 2009, ApJ, 690, 440 